\title{
DOA Estimation of Uncorrelated, Partly Correlated and Coherent Signals Using Alternating Oblique Projection
}

\author{
Huijun $H O U^{1}$, Xingpeng MAO ${ }^{1,2}$ \\ ${ }^{1}$ School of Electronics and Information Engineering, Harbin Institute of Technology, Harbin 150001, P. R. China \\ ${ }^{2}$ Collaborative Innovation Center of Information Sensing and Understanding, Harbin Institute of Technology, \\ Harbin 150001, P. R. China \\ mxp@hit.edu.cn
}

Submitted October 1, 2016 / Accepted February 16, 2017

\begin{abstract}
The problem of direction-of-arrival (DOA) estimation is important in array signal processing. To estimate the DOAs of uncorrelated, partly correlated and coherent signals, a new iterative DOA estimation algorithm, named AOP-DOA, is proposed by using alternating oblique projection (AOP). In each iteration, the oblique projection approach is employed to separate the received signals, then the DOA of each separated signal is estimated one after another. After theoretical analysis on the relationship between the proposed AOP-DOA and the conventional alternating projection based maximum likelihood estimator (AP-MLE), an $A O P \& A P-D O A$ algorithm, which is a combination of AOP$D O A$ and AP-MLE, is developed to reduce the computational complexity of AOP-DOA. Extensive experiments validate the effectiveness and complexity of the proposed two algorithms. Particularly, AOP\&AP-DOA keeps the merits of AOP-DOA, but exhibits superiority over AOP-DOA in terms of computational complexity when proper adaptive grid refinement strategy is applied.
\end{abstract}

\section{Keywords}

Oblique projection, direction-of-arrival (DOA) estimation, maximum likelihood (ML), coherent signal

\section{Introduction}

The problem of direction-of-arrival (DOA) estimation received considerable attention in the fields of radar, communication, sonar, etc. [1], [2]. The maximum likelihood (ML) [3-5] and subspace-based high resolution algorithms, including multiple signal classification (MUSIC) [6] and estimation of signal parameters via rotational invariance techniques (ESPRIT) [7], have been proved to be effective and computationally efficient for uncorrelated and partially correlated signals. The ML algorithms [3-5] are also capable of yielding asymptotically optimal solutions for fully correlated (coherent) signals, but they are computationally intensive since the multivariate nonlinear optimization procedure is involved [8]. The subspace-based MUSIC and ESPRIT are relatively computational simplicity and yield suboptimal solutions, but they are inefficient for coherent signals [9], [10].

To solve the aforementioned problems, several preprocessing algorithms, such as redundancy averaging [11], forward/backward spatial smoothing (FBSS) [12], [13], etc., are developed to decorrelate signals in the array covariance matrix. However, the algorithm of redundancy averaging leads to biased DOA estimates [14], and the FBSS algorithm suffers from performance degradation due to the array aperture loss [15], [16]. On the other hand, iterative algorithms, including the alternating projection (AP) technique [17], [18] and the modified Gauss-Newton technique [19] are investigated to circumvent the multivariate nonlinear optimization procedure. However, the global convergence of AP based ML estimator (AP-MLE) can not be guaranteed, and the Newtontype algorithms also have to be carefully initialized so that the global convergence is achieved [20].

Recently, much attention has been drawn to sparse signal reconstruction (SSR) perspective for DOA estimation [21]. Many SSR algorithms associated with $l_{p}$-normbased $(1 \geq p \geq 0)$ convex relaxation [22-25] and sparse Bayesian learning (SBL) [26], [27] are developed. They are capable of handling uncorrelated, partially correlated, and coherent signals, even if in the small sample-size case. Apart from the ML and SSR algorithms, the RELAX algorithm in [28] as well as the iterative adaptive approach for amplitude and phase estimation algorithm in [29] also works well in the same context.

In this paper, an alternating oblique projection (AOP) algorithm is proposed for DOA estimation. The technique of oblique projection (OP) [30] has a distinct advantage in terms of separating signal-of-interest (SOI) in the signal subspace while zero-forcing structured interferences in the interference subspace [31], [32]. In the context of DOA estimation, the OP technique was utilized to develop a scaled version of the MUSIC algorithm [33], and was utilized to integrate a prior known location of several sources into the MUSIC algorithm and circumvent their influences on the estimation 
of the unknown ones [34]. However, both are not applicable for coherent signals, since they aim at improving the performance of MUSIC for noncoherent signals. In [35-40], for DOA estimation of mixed coherent and noncoherent signals, the OP technique was utilized to eliminate the contributions of noncoherent signals from the data covariance matrix so that only those of coherent signals remain. Then the DOAs of coherent signals are estimated by the algorithm of spatial smoothing, redundancy averaging, etc.. But confined to the decorrelation algorithms, [35-40] were only researched with certain regular arrays (e.g., uniform linear array).

The proposed algorithm is named AOP-DOA, and is able to handle coherent signals. But different to [35-40], the proposed AOP-DOA has no limitation on antenna array geometry since the OP technique is differently used. Herein, AOP-DOA employs OP technique to alternately separate each of the received signals from array measurements. By this means, the received multiple signals are separated into a series of small signal groups, where each group contains one signal. Then the DOA of each signal is obtained by performing single target source localization. Based on AOPDOA, we also propose an AOP\&AP-DOA algorithm, which is a combination of AOP-DOA and AP-MLE, and reduces the computational complexity of AOP-DOA. follows.

The main contributions of this paper are presented as

1) By employing OP technique, a new AOP-DOA algorithm, which is applicable to arbitrary array geometries, is proposed for DOA estimation.

2) On the basis of AOP-DOA, an AOP\&AP-DOA algorithm, which could reduce the computational complexity of AOP-DOA, is proposed.

3) The proposed two algorithms can work well with uncorrelated and correlated (including coherent) signals.

The remainder of the paper is organized as follows. Section 2 describes the system model and introduces the basic assumptions. The derivation and discussion of AOP-DOA and AOP\&AP-DOA are presented in Sec. 3. In Sec. 4, simulation experiments are presented to evaluate the effectiveness of proposed algorithms, and performance comparisons between our algorithms and the existing algorithms are made. Finally, conclusions are drawn in Sec. 5.

Notations: matrices and vectors are denoted by boldface uppercase and lowercase letters, respectively. $(\cdot)^{\dagger},(\cdot)^{\mathrm{H}},\|\cdot\|$, $(\cdot)^{\perp}, E\{\cdot\}$ and $\operatorname{tr}\{\cdot\}$ denote the Moore-Penrose pseudoinverse, Hermitian transposition, $l_{2}$-norm, orthogonal complement, statistical expectation and trace operator, respectively. $(\cdot)^{-1}$ and eig $(\cdot)$ stand for the inverse and eigendecomposition of the bracketed matrix, respectively. Additionally, $\oplus$ represents the direct sum operator, $\mathcal{R}\{\cdot\}$ signifies the range space of the bracketed quantity, and $\hat{x}$ refers to the estimate of $x$.

\section{System Model and Assumptions}

Consider an antenna array composed of $M$ sensors with arbitrary array geometry, and assume that $K$ narrowband far-field source signals impinge on the array from distinct locations $\Theta_{1}, \Theta_{2}, \cdots, \Theta_{K}$. Further, the steering vector and the baseband signal waveform of the $k$ th incident signal are denoted as $\mathbf{a}\left(\Theta_{k}\right)$ and $s_{k}(t)$, respectively. $k=1,2, \cdots, K$, and the array output vector at the moment of $t$ can be expressed as [17], [41]

$$
\mathbf{x}(t)=\sum_{k=1}^{K} \mathbf{a}\left(\Theta_{k}\right) s_{k}(t)+\mathbf{n}(t)=\mathbf{A}(\boldsymbol{\Theta}) \mathbf{s}(t)+\mathbf{n}(t)
$$

where $\mathbf{A}(\boldsymbol{\Theta})=\left[\mathbf{a}\left(\Theta_{1}\right), \mathbf{a}\left(\Theta_{2}\right), \cdots, \mathbf{a}\left(\Theta_{K}\right)\right]$, and $\mathbf{s}(t)=$ $\left[s_{1}(t), s_{2}(t), \cdots, s_{K}(t)\right]^{\mathrm{T}} . \mathbf{n}(\mathbf{t})$ refers to the additive noise, which is modeled as a zero-mean, temporally and spatially white complex-valued Gaussian random process. Further, the covariance matrix of $\mathbf{n}(t)$ takes the form $\sigma^{2} \mathbf{I}$, where $\sigma^{2}$ signifies the noise power, and $\mathbf{I}$ denotes the identity matrix.

Using the system model (1), the data covariance matrix is given by

$$
\mathbf{R}_{x}=E\left\{\mathbf{x}(t) \mathbf{x}^{\mathrm{H}}(t)\right\}=\mathbf{A}(\boldsymbol{\Theta}) \mathbf{R}_{\mathbf{s}} \mathbf{A}^{\mathrm{H}}(\boldsymbol{\Theta})+\sigma^{2} \mathbf{I}
$$

where $\mathbf{R}_{\mathbf{s}}=E\left\{\mathbf{s}(t) \mathbf{S}^{\mathrm{H}}(t)\right\}$ denotes the source covariance matrix. Additionally, the following basic assumptions are made:

A1) The set of steering vectors $\left\{\mathbf{a}\left(\Theta_{1}\right), \mathbf{a}\left(\Theta_{2}\right), \cdots, \mathbf{a}\left(\Theta_{K}\right)\right\}$ is linearly independent for any set of distinct source locations $\Theta_{1}, \Theta_{2}, \cdots, \Theta_{K}$, where $K<M$.

A2) The narrowband signals $s_{1}(t), s_{2}(t), \cdots, s_{K}(t)$ are zeromean, and they may be uncorrelated, partially correlated or coherent. In addition, the signals are independent of the noise.

A3) The source number $K$ is known, or it has been estimated by the existing number detection methods (cf. [41], [42] and references therein).

The DOA estimation problem addressed in this paper is to estimate the source locations $\left\{\Theta_{k}\right\}_{k=1}^{K}$ from multiple snapshots $\left\{\mathbf{x}\left(t_{l}\right)\right\}_{l=1}^{L}$, where $L$ denotes the number of snapshots.

\section{Alternating Oblique Projection for DOA Estimation}

AOP-DOA is a data-dependent, alternating least squares (LS) adaptive algorithm based on the relaxed optimization principle ("one parameter at the time") in [43], [44]. The received signals are separated into multiple single signals by using OP technique, and the DOAs of the separated signals are estimated separately. To achieve source separation, the OP matrices in use are closely associated with the source 
locations. Because of this, AOP-DOA is implemented iteratively, and its cost function is given by [45]

$$
\frac{1}{L} \sum_{l=1}^{L}\left\|\mathbf{x}\left(t_{l}\right)-\sum_{k=1}^{K} \mathbf{a}\left(\Theta_{k}\right) s_{k}\left(t_{l}\right)\right\|^{2} .
$$

\subsection{Principle of AOP-DOA}

Assume that the signal at $\Theta_{k}$ is to be separated, where $k=1,2, \cdots, K$. Correspondingly, the signal $s_{k}(t)$ is referred to as the SOI, and the remaining $K-1$ received signals at $\left\{\Theta_{1}, \cdots, \Theta_{k-1}, \Theta_{k+1}, \cdots, \Theta_{K}\right\}$ are relatively referred to as the "interferences". Then, (1) can be rewritten as

$$
\mathbf{x}(t)=\mathbf{a}\left(\Theta_{k}\right) s_{k}(t)+\mathbf{B}_{k} \mathbf{S}_{\mathbf{B}_{k}}(t)+\mathbf{n}(t)
$$

where $\mathbf{s}_{\mathbf{B}_{k}}(t)=\left[s_{1}(t), \cdots, s_{k-1}(t), s_{k+1}(t), \cdots, s_{K}(t)\right]^{\mathrm{T}}$, and

$$
\mathbf{B}_{k}=\left[\mathbf{a}\left(\Theta_{1}\right), \cdots, \mathbf{a}\left(\Theta_{k-1}\right), \mathbf{a}\left(\Theta_{k+1}\right), \cdots, \mathbf{a}\left(\Theta_{K}\right)\right] .
$$

According to the basic assumptions claimed in Sec. 2, the signal subspace is given by $\mathcal{R}\{\mathbf{A}(\boldsymbol{\Theta})\}=\mathcal{R}\left\{\mathbf{a}\left(\Theta_{k}\right)\right\} \oplus$ $\mathcal{R}\left\{\mathbf{B}_{k}\right\}$, where $\mathcal{R}\left\{\mathbf{a}\left(\Theta_{k}\right)\right\}$ and $\mathcal{R}\left\{\mathbf{B}_{k}\right\}$ are disjoint. Therefore, the OP matrix whose range space is $\mathcal{R}\left\{\mathbf{B}_{k}\right\}$ and whose null space contains $\mathcal{R}\left\{\mathbf{a}\left(\Theta_{k}\right)\right\}$ can be expressed as [32]

$$
\mathbf{E}_{\mathbf{B}_{k}, \mathbf{a}\left(\Theta_{k}\right)}=\mathbf{B}_{k}\left(\mathbf{P}_{\mathbf{a}\left(\Theta_{k}\right)}^{\perp} \mathbf{B}_{k}\right)^{\dagger}
$$

where $\mathbf{P}_{\mathbf{a}\left(\Theta_{k}\right)}^{\perp}=\mathbf{I}-\mathbf{a}\left(\Theta_{k}\right) \mathbf{a}^{\dagger}\left(\Theta_{k}\right), \mathbf{E}_{\mathbf{B}_{k}, \mathbf{a}\left(\Theta_{k}\right)} \mathbf{a}\left(\Theta_{k}\right)=\mathbf{0}$, and $\mathbf{E}_{\mathbf{B}_{k}, \mathbf{a}\left(\Theta_{k}\right)} \mathbf{B}_{k}=\mathbf{B}_{k}$. Naturally, the received SOI can be separated from the array measurements in the following way:

$$
\mathbf{y}_{k}(t)=\left(\mathbf{I}-\mathbf{E}_{\mathbf{B}_{\mathbf{k}}, \mathbf{a}\left(\Theta_{\mathbf{k}}\right)}\right) \mathbf{z}(\mathbf{t})
$$

where

$$
\mathbf{z}(t)=\mathbf{x}(t)-\mathbf{n}(t)
$$

signifies the array output in noiseless scenario, and $\mathbf{y}_{k}(t)$ is referred to as the "cleaned" observation of the SOI.

Based on (4), (7) and the LS criterion in [46], the SOI can be finally estimated by solving the following minimization problem:

$$
\min _{\Theta_{k},\left\{s_{k}\left(t_{l}\right)\right\}_{l=1}^{L}} \frac{1}{L} \sum_{l=1}^{L}\left\|\mathbf{y}_{k}\left(t_{l}\right)-\mathbf{a}\left(\Theta_{k}\right) s_{k}\left(t_{l}\right)\right\|^{2} .
$$

Minimizing (9) with respect to $s_{k}\left(t_{l}\right)$, then the optimal solution to this problem yields

$$
\hat{s}_{k}\left(t_{l}\right)=\mathbf{a}^{\dagger}\left(\Theta_{k}\right) \mathbf{y}_{k}\left(t_{l}\right), \quad l=1,2, \cdots, L .
$$

Substituting (10) into (9), it follows that the DOA of the SOI can be estimated by solving the following minimization problem:

$$
\hat{\Theta}_{k}=\underset{\Theta_{k}}{\operatorname{argmin}} \frac{1}{L} \sum_{l=1}^{L}\left\|\mathbf{P}_{\mathbf{a}\left(\Theta_{k}\right)}^{\perp} \mathbf{y}_{k}\left(t_{l}\right)\right\|^{2} .
$$

In the above manner, all the $K$ received signals can be separated one after another, and the DOA estimate of each separated signal can be achieved separately. Remark that the OP matrix in (7) is irrelevant to the correlations among signals, and it is this property that makes the proposed AOP-DOA being applicable for uncorrelated, partially correlated and coherent signals. But it is noted that the computation of $\mathbf{E}_{\mathbf{B}_{k}, \mathbf{a}\left(\Theta_{k}\right)}$ requires knowledge of the source locations, whereas none of them are considered to be known a prior. To solve this problem, AOP-DOA is iterative, and it updates $\hat{\Theta}_{k}^{(i)}$ by using previously estimated values $\left\{\hat{\Theta}_{1}^{(i)}, \cdots, \hat{\Theta}_{k-1}^{(i)}, \hat{\Theta}_{k}^{(i-1)}\right.$, $\left.\hat{\Theta}_{k+1}^{(i-1)}, \cdots, \hat{\Theta}_{K}^{(i-1)}\right\}$, where $\hat{\Theta}_{k}^{(i)}$ denotes the DOA estimates of $\Theta_{k}$ at the $i$ th iteration (similarly hereinafter), $i=1,2, \cdots$.

The relative change of the cost function in (3) between two consecutive iterations determines the convergence of AOP-DOA. Substituting the iteration results of (10) and (11) into (3), it follows that (see the Appendix A)

$$
\begin{aligned}
& \frac{1}{(M-K) L} \sum_{l=1}^{L}\left\|\mathbf{x}\left(t_{l}\right)-\sum_{k=1}^{K} \mathbf{a}\left(\Theta_{k}\right) s_{k}\left(t_{l}\right)\right\|^{2}-\frac{K}{M-K} \hat{\sigma}^{2} \\
& =\frac{1}{M-K} \operatorname{tr}\left\{\mathbf{P}_{\mathbf{A}(\Theta)}^{\perp} \hat{\mathbf{R}}_{x}\right\}=\hat{\sigma}^{2}
\end{aligned}
$$

where $\hat{\mathbf{R}}_{x}=\frac{1}{L} \sum_{l=1}^{L} \mathbf{x}\left(t_{l}\right) \mathbf{x}^{\mathrm{H}}\left(t_{l}\right)$ is referred to as the sample data covariance matrix which signifies a practical estimation of the data covariance matrix from the samples $\left\{\mathbf{x}\left(t_{l}\right)\right\}_{l=1}^{L}$. $\hat{\sigma}^{2}=\frac{1}{L} \sum_{l=1}^{L}\left\|\mathbf{n}\left(t_{l}\right)\right\|^{2}$ denotes an estimation of noise power from the samples $\left\{\mathbf{n}\left(t_{l}\right)\right\}_{l=1}^{L}$.

Instead of using (3), it is natural to terminate the AOPDOA iterations by checking the relative change of

$$
\frac{1}{M-K} \operatorname{tr}\left\{\mathbf{P}_{\mathbf{A}(\Theta)}^{\perp} \hat{\mathbf{R}}_{x}\right\}
$$

The fundamental objective of the proposed AOP-DOA is to find the global optimal solution of the nonlinear LS problem in (3), and it is achieved by separately solving the optimization problem of a series of separable variable pairs $\left\{\Theta_{k}, s_{k}(t)\right\}_{k=1}^{K}($ see $(9))$ and following the relaxed iterative approach in [28] (the iterative implementation of AOP-DOA is summarized in Tab. 1, Sec. 3.2). These processes are essentially the same as these of the RELAX estimator in [28], hence the convergence properties of AOP-DOA and RELAX are consistent.

The same as RELAX, for the proposed AOP-DOA, the estimation results of "interferences" are removed from the array measurements, and the parameters of the SOI are estimated based on the cleaned data. The difference of these two algorithms is that AOP-DOA obtain the "cleaned" observation of the SOI from noiseless array output via employing the OP technique, whereas that of RELAX is obtained by directly subtracting the "interferences" from the noisy array measurements. Because of this, the specific implementation of AOP-DOA is quite distinct from that of RELAX (cf. [28]), which will be described in the following subsection. 


\subsection{Implementation of AOP-DOA}

According to Sec. 3.1, at the $i$ th iteration, $\mathbf{y}_{k}(t)$ is calculated as follows:

$$
\mathbf{y}_{k}^{(i)}(t)=\left(\mathbf{I}-\mathbf{E}_{\hat{\mathbf{B}}_{k}^{(i)}, \mathbf{a}\left(\hat{\Theta}_{k}^{(i-1)}\right)}\right) \mathbf{z}(t)
$$

where

$$
\hat{\mathbf{B}}_{k}^{(i)}=\left[\mathbf{a}\left(\hat{\Theta}_{1}^{(i)}\right), \cdots, \mathbf{a}\left(\hat{\Theta}_{k-1}^{(i)}\right), \mathbf{a}\left(\hat{\Theta}_{k+1}^{(i-1)}\right), \cdots, \mathbf{a}\left(\hat{\Theta}_{K}^{(i-1)}\right)\right] .
$$

Further, it follows from (8) and (14) that

$$
\begin{aligned}
\hat{\mathbf{R}}_{y_{k}}^{(i)} & =\frac{1}{L} \sum_{l=1}^{L} \mathbf{y}_{k}^{(i)}\left(t_{l}\right)\left(\mathbf{y}_{k}^{(i)}\left(t_{l}\right)\right)^{\mathrm{H}} \\
& =\left(\mathbf{I}-\mathbf{E}_{\hat{\mathbf{B}}_{k}^{(i)}, \mathbf{a}\left(\hat{\Theta}_{k}^{(i-1)}\right)}\right) \hat{\mathbf{R}}_{z}\left(\mathbf{I}-\mathbf{E}_{\hat{\mathbf{B}}_{k}^{(i)}, \mathbf{a}\left(\hat{\Theta}_{k}^{(i-1)}\right)}\right)^{\mathrm{H}} .
\end{aligned}
$$

where

$$
\hat{\mathbf{R}}_{z}=\frac{1}{L} \sum_{l=1}^{L} \mathbf{z}\left(t_{l}\right) \mathbf{z}^{\mathrm{H}}\left(t_{l}\right)=\hat{\mathbf{R}}_{x}-\hat{\sigma}^{2} \mathbf{I} .
$$

Thus, (11) can be rewritten as

$$
\hat{\Theta}_{k}^{(i)}=\underset{\Theta_{k}}{\operatorname{argmin}} \operatorname{tr}\left\{\mathbf{P}_{\mathbf{a}\left(\Theta_{k}\right)}^{\perp} \hat{\mathbf{R}}_{y_{k}}^{(i)}\right\} .
$$

Note that $\operatorname{tr}\left\{\mathbf{P}_{\mathbf{a}\left(\Theta_{k}\right)}^{\perp} \hat{\mathbf{R}}_{y_{k}}^{(i)}\right\}=\operatorname{tr}\left\{\hat{\mathbf{R}}_{y_{k}}^{(i)}\right\}-\operatorname{tr}\left\{\mathbf{P}_{\mathbf{a}\left(\Theta_{k}\right)} \hat{\mathbf{R}}_{y_{k}}^{(i)}\right\}$. Hence, the optimal problem of (18) is equivalent to

$\hat{\Theta}_{k}^{(i)}=\underset{\Theta_{k}}{\operatorname{argmax}} \operatorname{tr}\left\{\mathbf{P}_{\mathbf{a}\left(\Theta_{k}\right)} \hat{\mathbf{R}}_{y_{k}}^{(i)}\right\}=\underset{\Theta_{k}}{\operatorname{argmax}} \frac{\mathbf{a}^{\mathrm{H}}\left(\Theta_{k}\right) \hat{\mathbf{R}}_{y_{k}}^{(i)} \mathbf{a}\left(\Theta_{k}\right)}{\mathbf{a}^{\mathrm{H}}\left(\Theta_{k}\right) \mathbf{a}\left(\Theta_{k}\right)}$.

Using Rayleigh quotient properties in [47], [48], the solution to this problem yields

$$
\mathcal{R}\left\{\mathbf{a}\left(\hat{\Theta}_{k}^{(i)}\right\}=\mathcal{R}\left\{\mathbf{u}_{\max , k}^{(i)}\right\}\right.
$$

where $\mathbf{u}_{\max , k}^{(i)}$ refers to the unit eigenvector corresponding to the maximum eigenvalue of $\hat{\mathbf{R}}_{y_{k}}^{(i)}$. Therefore,

$$
\hat{\Theta}_{k}^{(i)}=\underset{\Theta_{k}}{\operatorname{argmax}}\left|\mathbf{a}^{\mathrm{H}}\left(\Theta_{k}\right) \mathbf{u}_{\max , k}^{(i)}\right|^{2} .
$$

This maximization problem can be solved without much computational effort by means of adaptive grid refinement [23] only around the regions where signals were present in the last iteration.

Using (16), (21) and referring to the relaxed iterative method in [28], the implementation procedure of AOP-DOA is summarized in Tab. 1, where $\mathcal{S}^{(0)}$ represents a rough region of potential source locations (a $1^{\circ}$ or $2^{\circ}$ uniform sampling usually suffices [23]), and $\mathcal{S}_{j}^{(i)}$ denotes a refined region around the previous source location $\hat{\Theta}_{j}^{(i-1)}, j=1,2, \cdots, K$.

As given in Tab. 1, the initialization step of AOPDOA is implemented with assumption $\mathbf{a}\left(\hat{\Theta}_{k}^{(-1)}\right)=\mathbf{0}$, where $k=1,2, \cdots, K$. At the beginning of each outer loop, $\hat{\Theta}_{k}^{(0)}$ is determined with (16) and (21) (see lines 5-7). After that, we sequentially redetermine $\hat{\Theta}_{1}, \hat{\Theta}_{2}, \cdots, \hat{\Theta}_{k}$ and iteratively update these DOA estimates until convergence is achieved (see lines 8-18). AOP-DOA stops when all of the $K$ outer loops finished.

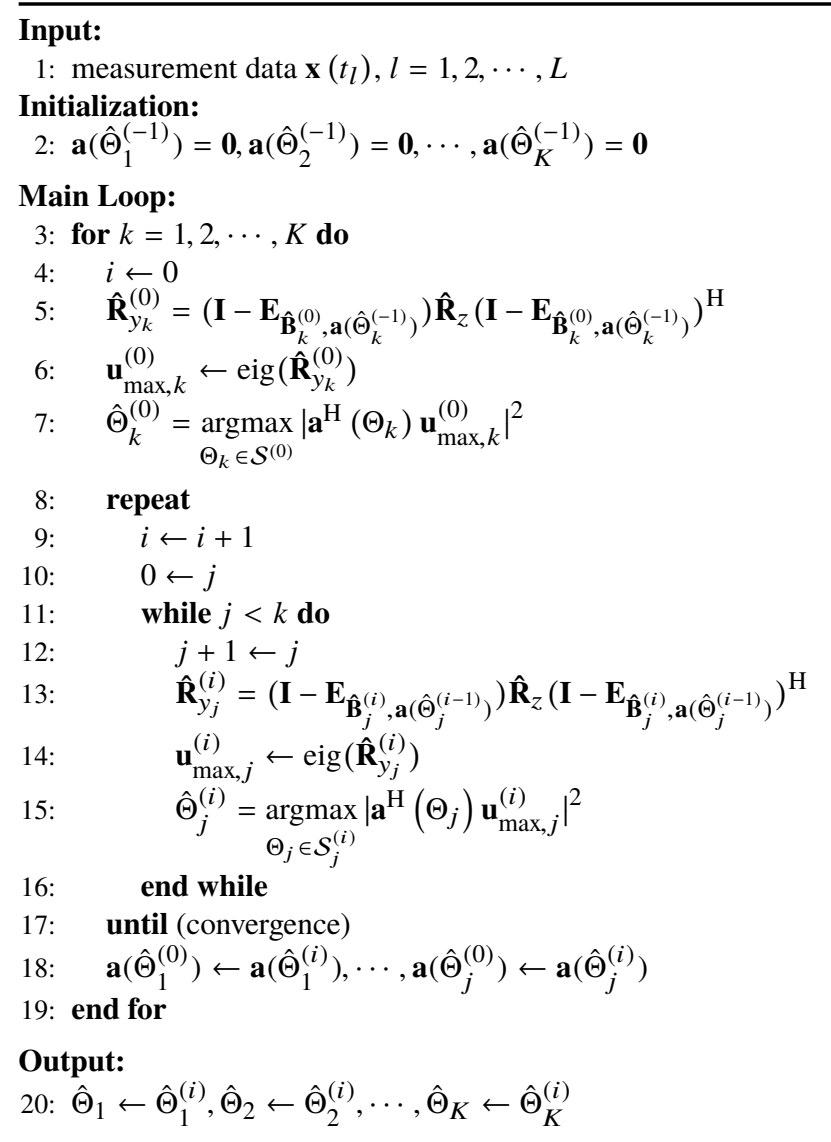

Tab. 1. The AOP-DOA algorithm.

\subsection{Relationship Between AOP-DOA and AP-MLE}

At the $i$ th iteration, AP-MLE updates the $k$ th DOA estimates as follows [17]:

$$
\begin{aligned}
& \hat{\Theta}_{k, \text { AP-MLE }}^{(i)}=\underset{\Theta_{k}}{\operatorname{argmin}} \operatorname{tr}\left\{\mathbf{P}_{\left[\tilde{\mathbf{B}}_{k}^{(i-1)}, \mathbf{a}\left(\Theta_{k}\right)\right]}^{\perp} \hat{\mathbf{R}}_{x}\right\} \\
& =\underset{\Theta_{k}}{\operatorname{argmin}} \operatorname{tr}\left\{\mathbf{P}_{\left[\tilde{\mathbf{B}}_{k}^{(i-1)}, \mathbf{a}\left(\Theta_{k}\right)\right]}^{\perp} \hat{\mathbf{R}}_{z}+\hat{\sigma}^{2} \mathbf{P}_{\left[\tilde{\mathbf{B}}_{k}^{(i-1)}, \mathbf{a}\left(\Theta_{k}\right)\right]}^{\perp}\right\} \\
& =\underset{\Theta_{k}}{\operatorname{argmin}} \operatorname{tr}\left\{\mathbf{P}_{\left[\tilde{\mathbf{B}}_{k}^{(i-1)}, \mathbf{a}\left(\Theta_{k}\right)\right]}^{\perp \mathbf{R}_{z}}+\hat{\sigma}^{2}(M-K)\right\}
\end{aligned}
$$

where $\tilde{\mathbf{B}}_{k}^{(i)}=\left[\mathbf{a}\left(\hat{\Theta}_{1}^{(i)}\right), \cdots, \mathbf{a}\left(\hat{\Theta}_{k-1}^{(i)}\right), \mathbf{a}\left(\hat{\Theta}_{k+1}^{(i)}\right), \cdots, \mathbf{a}\left(\hat{\Theta}_{K}^{(i)}\right)\right]$. According to Appendix B, $\mathbf{E}_{\mathbf{a}\left(\Theta_{k}\right), \mathbf{B}_{k}}=\mathbf{P}_{\mathbf{a}\left(\Theta_{k}\right)}\left(\mathbf{I}-\mathbf{E}_{\mathbf{B}_{k}, \mathbf{a}\left(\Theta_{k}\right)}\right)$ and

$$
\begin{aligned}
\mathbf{P}_{\mathbf{A}(\Theta)}^{\perp} & =\mathbf{I}-\mathbf{E}_{\mathbf{B}_{k}, \mathbf{a}\left(\Theta_{k}\right)}-\mathbf{P}_{\mathbf{a}\left(\Theta_{k}\right)}\left(\mathbf{I}-\mathbf{E}_{\mathbf{B}_{k}, \mathbf{a}\left(\Theta_{k}\right)}\right) \\
& =\mathbf{P}_{\mathbf{a}\left(\Theta_{k}\right)}^{\perp}\left(\mathbf{I}-\mathbf{E}_{\mathbf{B}_{k}, \mathbf{a}\left(\Theta_{k}\right)}\right) .
\end{aligned}
$$

Therefore, (22) is equivalent to

$$
\hat{\Theta}_{k, \text { AP-MLE }}^{(i)}=\underset{\Theta_{k}}{\operatorname{argmin}} \sum_{l=1}^{L} \frac{\left\|\mathbf{P}_{\mathbf{a}\left(\Theta_{k}\right)}^{\perp}\left(\mathbf{I}-\mathbf{E}_{\tilde{\mathbf{B}}_{k}^{(i-1)}, \mathbf{a}\left(\Theta_{k}\right)}\right) \mathbf{z}(t)\right\|^{2}}{L} .
$$

Recall from (16) and (18) that, at the $i$ th iteration, AOPDOA updates the $k$ th DOA estimate as follows: 


\section{Input:}

1: $\left\{\hat{\Theta}_{k}\right\}_{k=1}^{K}$ : DOA estimates obtained from AOP-DOA

\section{Initialization:}

2: $\hat{\Theta}_{1}^{(0)} \leftarrow \hat{\Theta}_{1}, \hat{\Theta}_{2}^{(0)} \leftarrow \hat{\Theta}_{2}, \cdots, \hat{\Theta}_{K}^{(0)} \leftarrow \hat{\Theta}_{K}$

Main Loop:

3: $i \leftarrow 0$

4: repeat

5: $\quad i \leftarrow i+1$

6: $\quad$ for $k=1,2, \cdots, K$ do

7: $\quad \hat{\Theta}_{k}^{(i)}=\underset{\Theta_{k} \in \mathcal{S}_{k}^{(i)}}{\operatorname{argmin}} \operatorname{tr}\left\{\mathbf{P}_{\left[\tilde{\mathbf{B}}_{k}^{(i-1)}, \mathbf{a}\left(\Theta_{k}\right)\right]}^{\perp} \hat{\mathbf{R}}_{x}\right\}$

8: $\quad$ end for

9: until (convergence)

\section{Output:}

10: $\hat{\Theta}_{1}=\hat{\Theta}_{1}^{(i)}, \hat{\Theta}_{2}=\hat{\Theta}_{2}^{(i)}, \cdots, \hat{\Theta}_{K}=\hat{\Theta}_{K}^{(i)}$

Tab. 2. The AOP\&AP-DOA algorithm.

$$
\begin{aligned}
& \hat{\Theta}_{k, \text { AOP-DOA }}^{(i)}=\underset{\Theta_{k}}{\operatorname{argmin}} \operatorname{tr}\left\{\mathbf{P}_{\mathbf{a}\left(\Theta_{k}\right)}^{\perp} \hat{\mathbf{R}}_{y_{k}}^{(i)}\right\} \\
& =\underset{\Theta_{k}}{\operatorname{argmin}} \frac{1}{L} \sum_{l=1}^{L} \| \mathbf{P}_{\mathbf{a}\left(\Theta_{k}\right)}^{\perp}\left(\mathbf{I}-\mathbf{E}_{\left.\hat{\mathbf{B}}_{k}^{(i)}, \mathbf{a}\left(\hat{\Theta}_{k}^{(i-1)}\right)\right) \mathbf{z}(t) \|^{2} .}\right.
\end{aligned}
$$

Evidently, both AOP-DOA and AP-MLE solve onedimensional optimal problems. Comparing (24) with (25), it is found that AOP-DOA utilizes previously estimated value $\hat{\Theta}_{k}^{(i-1)}$ to estimate $\hat{\Theta}_{k}^{(i)}$, whereas AP-MLE does not.

The results of AOP-DOA can be used to provide a good initial estimate for the main loop of AP-MLE. This method is referred to as AOP\&AP-DOA and is summarized in Tab. 2. Instead of adopting a universally fine grid search, the source locations can be accurately estimated via AP-MLE by means of a fine search only around the estimated values of AOPDOA. By this means, AOP-DOA provides relatively coarse DOA estimates in the first stage (e.g., in the adaptive gird refinement strategy, the lower limit of fine gird spacing (LLFGS) may be set to $0.1^{\circ}, 0.25^{\circ}$, etc.), and, in the second stage, AP-MLE provides fine DOA estimates (e.g., the LLFGS can be set to $0.01^{\circ}$ ).

\subsection{Computational Complexity}

The computational complexity of proposed AOP-DOA is briefly analyzed as follows, where a flop is defined as a complex floating-pint multiplication or addition operation.

The computation of matrix $\hat{\mathbf{R}}_{z}$ takes about $O\left(L M^{2}+\right.$ $M^{3}$ ) flops, where the noise power estimate $\hat{\sigma}^{2}$ is obtained by averaging the $M-K$ smallest eigenvalues of covariance matrix $\hat{\mathbf{R}}_{x}$ [6]. The calculation of the OP matrix $\mathbf{E}_{\hat{\mathbf{B}}_{k}^{(i)}, \mathbf{a}\left(\hat{\Theta}_{k}^{(i-1)}\right)}$ (or $\left.\mathbf{E}_{\hat{\mathbf{B}}_{j}^{(i)}, \mathbf{a}\left(\hat{\Theta}_{j}^{(i-1)}\right)}\right)$ requires roughly $O\left((K-1) M^{2}\right)$ flops, and that of the matrix $\hat{\mathbf{R}}_{y_{k}}^{(i)}$ (or $\hat{\mathbf{R}}_{y_{j}}^{(i)}$ ) requires roughly $O\left(M^{3}\right)$ flops. The eigendecomposition of $\hat{\mathbf{R}}_{y_{k}}^{(i)}$ (or $\hat{\mathbf{R}}_{y_{j}}^{(i)}$ ) requires approxi- mately $O\left(M^{3}\right)$ flops. Suppose that $\mathcal{S}^{(0)}$ and $\mathcal{S}_{j}^{(i)}$ consist of $N_{0}$ and $N_{i}$ potential source locations, respectively. Then the number of flops roughly required to solve the maximization problem in line 7, Tab. 1 and line 15, Tab. 1 is $O\left(M N_{0}\right)$ and $O\left(M N_{i}\right)$, respectively. In addition, the computation of the cost function in (13) requires roughly $O\left(M^{3}\right)$ flops.

Therefore, the computational complexity of AOP-DOA is roughly $O\left(L M^{2}+M^{3}+K\left(2 M^{3}+(K-1) M^{2}+M N_{0}\right)+\right.$ $\left.\sum_{k=1}^{K} \sum_{i=1}^{n_{k}}\left(\left(2 M^{3}+(K-1) M^{2}+M N_{i}\right) k+M^{3}\right)\right)$ in total, where $n_{k}$ denotes the iteration number included in the $k$ th outer loop. In particular, the total computational complexity is approximately $O\left(L M^{2}+K\left(M^{3}+M N_{0}\right)+\sum_{k=1}^{K} \sum_{i=1}^{n_{k}}\left(M^{3}+\right.\right.$ $\left.M N_{i}\right) k$ ) flops, when $N_{0} \gg M>K$, which occurs often in practical applications.

The proposed AOP\&AP-DOA can be viewed as a combination of AOP-DOA and AP-MLE. Thus the computational complexity of AOP\&AP-DOA consists of two parts, where the part of AP-MLE additionally takes about $O\left(M^{3} K \tilde{N}_{i} n_{A P}\right)$ flops. $n_{A P}$ denotes the iteration number, and it is supposed that $\mathcal{S}_{k}^{(i)}$ (line 7, Tab. 2) consists of $\tilde{N}_{i}$ potential source locations.

The computational complexities of AOP-DOA and AOP\&AP-DOA are summarized in Tab. 3. Remark that the major computational complexity is proportional to $\sum_{i=1}^{n_{k}} N_{i}$ and $N_{0}$, when $M, K$ and $L$ are fixed. Therefore, the employed adaptive grid refinement strategy not only determines the computational accuracy, but also influences the computational cost. Compared with AOP-DOA, in Sec. 4, we show that AOP\&AP-DOA could have a lower computational load when proper adaptive grid refinement strategy is applied.

\begin{tabular}{|c|c|}
\hline Algorithm & Complexity \\
\hline AOP-DOA & $O\left(L M^{2}+K\left(M^{3}+M N_{0}\right)\right)$ \\
& $+O\left(\sum_{k=1}^{K} \sum_{i=1}^{n_{k}}\left(M^{3}+M N_{i}\right) k\right)$ \\
\hline AOP\&AP-DOA & $\begin{array}{c}O\left(M^{3} K \tilde{N}_{i} n_{A P}+L M^{2}+K\left(M^{3}+M N_{0}\right)\right) \\
+O\left(\sum_{k=1}^{K} \sum_{i=1}^{n_{k}}\left(M^{3}+M N_{i}\right) k\right)\end{array}$ \\
\hline
\end{tabular}

Tab. 3. Computational complexity of the proposed algorithms.

\section{Simulation Results}

Extensive experiments are presented to evaluate the effectiveness of proposed AOP-DOA and AOP\&AP-DOA. The stochastic Cramér-Rao bound (CRB) [3] and the existing high resolution DOA estimation algorithms, including AP-MLE [17], RELAX [28], MUSIC [6], $l_{1}$-SVD [23] and perturbed SBL (PSBL) [27] are utilized for performance comparison.

The root mean square error (RMSE) of DOA estimates is defined as

$$
\mathrm{RMSE}=\sqrt{\frac{1}{K M_{c}} \sum_{m=1}^{M_{c}} \sum_{k=1}^{K}\left(\hat{\Theta}_{m, k}-\Theta_{k}\right)^{2}}
$$


where $M_{c}$ is the number of trials, $\hat{\Theta}_{m, k}$ denotes the estimate of the $k$ th DOA $\Theta_{k}$ in the $m$ th trial. All the simulations are carried out on a uniform linear array with $M=6$ sensors and half-wavelength sensor spacing. Unless otherwise stated, each of the simulations is evaluated by $M_{c}=200$ independent trials, and $L=100$ snapshots are employed.

The initialization condition for PSBL is set in the same way as [27]. $l_{1}$-SVD is considered with SeDuMi packages [49], and the regularization parameter is set to 0.625 . For $l_{1}$-SVD, RELAX, AOP-DOA and AOP\&AP-DOA, the adaptive grid refinement strategy [23] is employed, where the spacing of the initial coarse grid is $2^{\circ}$ and the LLFGS is set to $0.01^{\circ}$, unless otherwise stated. For MUSIC and AP-MLE, we use $0.01^{\circ}$ uniform sampling of the spatial location of the sources. All the simulations are performed using MATLAB 2013b running on a computer with a $2.3 \mathrm{GHz}$ Intel Quad-Core processor and 12GB RAM, under Windows 8.1. The computational complexity of an algorithm is evaluated in terms of the average CPU processing time [27].

\subsection{Discussion: Complexity and Accuracy}

In Sec. 3.4, the complexities of the proposed algorithms are analyzed, and it is shown that, when the number of snapshots $L$, array size $M$ and source number $K$ are fixed, the computational complexity is jointly proportional to the iteration number and the grid size which is considered in the adaptive grid refinement strategy.

This subsection intends to evaluate the complexities of proposed AOP-DOA and AOP\&AP-DOA by employing different adaptive grid refinement strategies. Two equal-power uncorrelated signals impinging from $\left[-5.12^{\circ}, 5.37^{\circ}\right]$ are considered in this experiment, and the RMSE is utilized to evaluate the precision of DOA estimates. Both the CPU time and the average number of iterations are analyzed at each evaluated signal-to-noise ratio (SNR). In each simulation, the number of iterations of AOP\&AP-DOA is the sum of the iteration numbers that required in the two stages DOA estimation.

Firstly, the proposed AOP-DOA is investigated. Three different LLFGS are considered, and the SNR is varied from $-15 \mathrm{~dB}$ to $25 \mathrm{~dB}$. Fig. 1 shows the statistical results. It is seen that AOP-DOA exhibits a satisfying performance when different LLFGS is used. Moreover, AOP-DOA can coincide well with the CRB at a moderate to high SNR when proper adaptive grid refinement strategy is employed. But we also should note that a fine grid search requires a heavy computational cost.

In the next, the proposed AOP\&AP-DOA is evaluated. Since the DOA estimation of AOP\&AP-DOA consists of two stages, we consider different LLFGS in the first stage. But the LLFGS in the second stage is fixed at $0.01^{\circ}$. Other settings remain the same as previous experiment. Figure 2 shows the statistical results of AOP\&AP-DOA, and it is indicated that AOP\&AP-DOA is able to coincide well with the CRB at a moderate to high SNR. But AOP\&AP-DOA consumes different time, when different adaptive grid refinement strategies are applied. The running time is proportional to the LLFGS in the first stage. With proper adaptive grid refinement strategy, the convergence speed of AOP\&AP-DOA is fast. In the following simulations, the LLFGS in the first stage of AOP\&AP-DOA is fixed at $0.1^{\circ}$ and that in the second stage is fixed at $0.01^{\circ}$.

Besides, when comparing Fig. 1 with Fig. 2, it can be seen that AOP-DOA may show a competitive estimation precision with a high SNR, but AOP\&AP-DOA can own a lower running time. In particular, a fine grid search leads to a large number of iterations. But for a fixed grid search approach, with the increase of SNR, the numbers of iterations of AOP-DOA and AOP\&AP-DOA are stable or approximately stable, especially when the incident signals dominate the background noise. The same is true for the running time under different SNR.

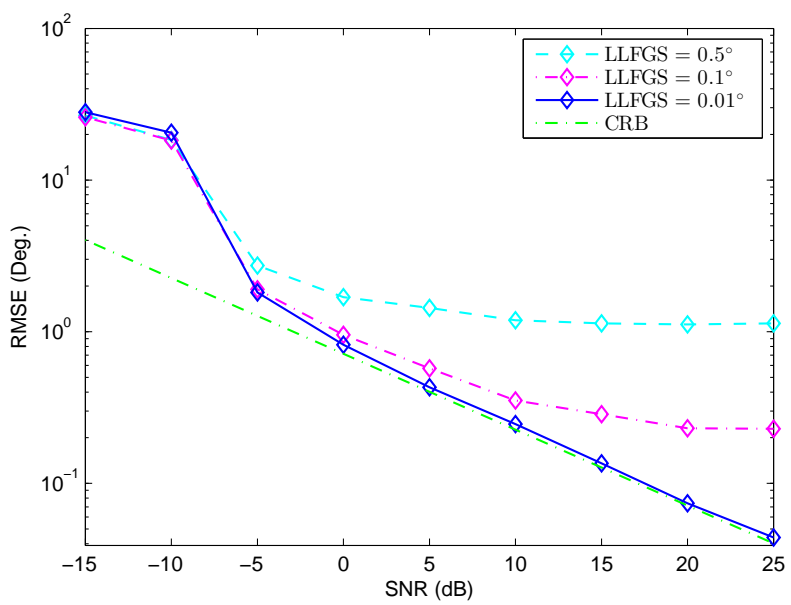

(a) RMSE versus SNR

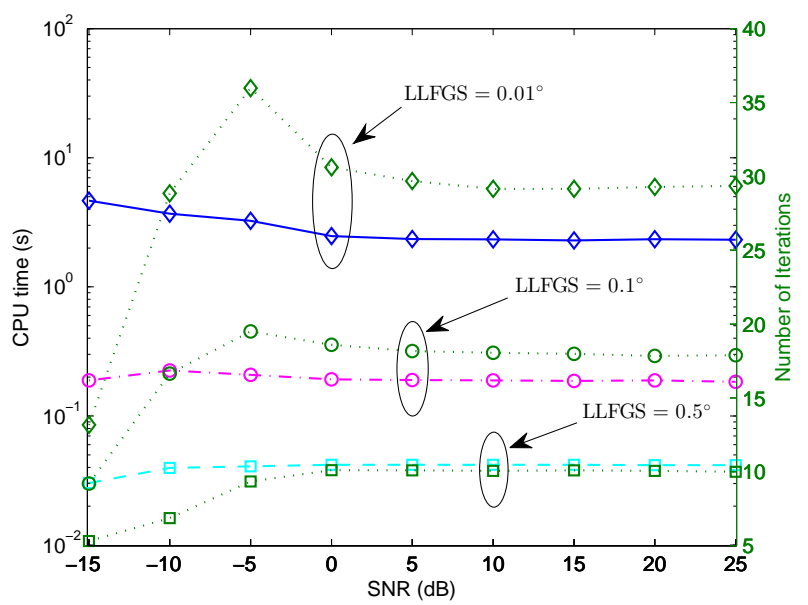

(b) CPU time versus SNR

Fig. 1. RMSE and CPU time comparison of the proposed AOP-DOA for two uncorrelated sources impinging from $\left[-5.12^{\circ}, 5.37^{\circ}\right]$ when three different LLFGS are considered. The SNR ranges from $-15 \mathrm{~dB}$ to $25 \mathrm{~dB}$. 


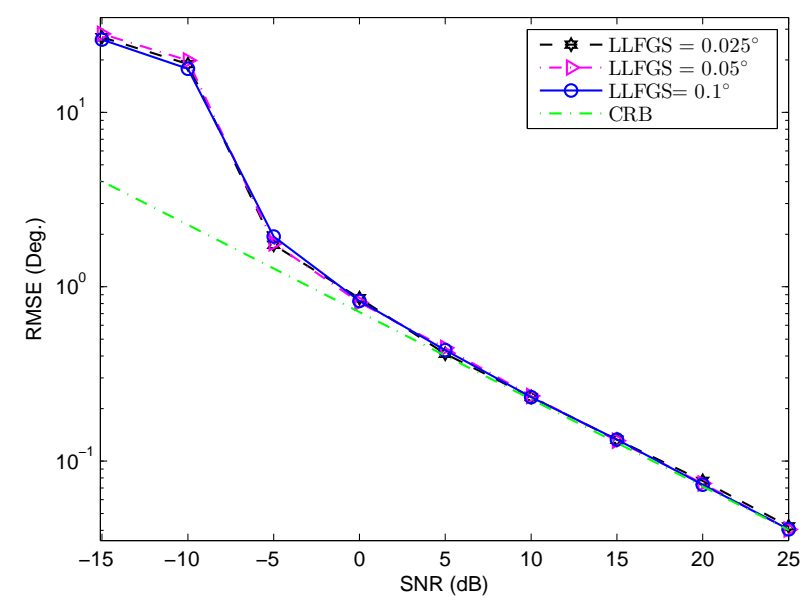

(a) RMSE versus SNR

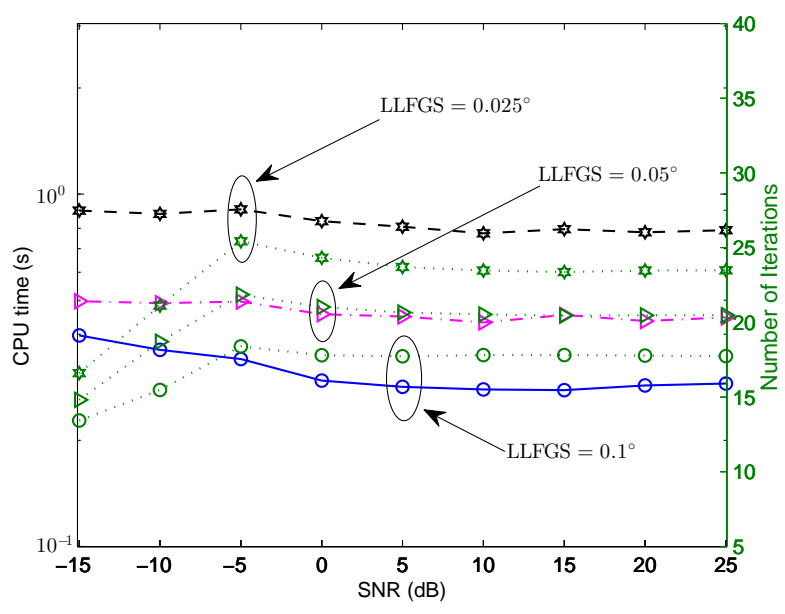

(b) CPU time versus SNR

Fig. 2. RMSE and CPU time comparison of the proposed AOP\&AP-DOA for two uncorrelated sources impinging from $\left[-5.12^{\circ}, 5.37^{\circ}\right]$ when the LLFGS in the first stage ranges from $0.025^{\circ}$ to $0.1^{\circ}$. The LLFGS in the second stage is fixed at $0.01^{\circ}$, and the SNR ranges from $-15 \mathrm{~dB}$ to $25 \mathrm{~dB}$.

\subsection{RMSE and CPU Time Comparisons}

This subsection presents detailed performance analyses to illustrate the superiority of the proposed AOP-DOA and AOP\&AP-DOA in cases of uncorrelated and correlated(including coherent) signals. For each algorithm, the computational complexity and the estimation accuracy are employed for performance comparison, and they are evaluated by the running time and the RMSE, respectively.

Figure 3 depicts the estimation accuracy of the DOA estimates and the time usage of each comparing algorithm when two uncorrelated signals are considered. We assume the signals impinge on the array from $\left[-5.12^{\circ}, 5.37^{\circ}\right]$, and they have equal SNR ranging from $-15 \mathrm{~dB}$ to $20 \mathrm{~dB}$. The results of Fig. 3(a) show that the proposed AOP-DOA and AOP\&AP-DOA, as well as RELAX and AP-MLE, exhibit a satisfying estimation precision, whereas PSBL and $l_{1}-\mathrm{SVD}$ not coincide well with the CRB. It is of interest to note that MUSIC also shows a high precision when the SNR is high enough, but it fails to hold a superresolution at a lower SNR. Fig. 3(b) indicates that AP-MLE consumes the maximum running time, while the proposed AOP-DOA and AOP\&APDOA algorithms take the relatively lower one. This is because the conventional AP-MLE always adopts a universally fine grid search, whereas our algorithms utilize fine grid search only around the regions where signals are present. Besides, the computational load of RELAX and the proposed AOP-DOA is approximately at the same level, and is higher than that of the proposed AOP\&AP-DOA. The running time of PSBL fluctuates remarkably when the SNR changes, but that of the other algorithms are not. To illustrate this further, Fig. 3(c) presents the iteration numbers of PSBL, RELAX and the proposed AOP-DOA and AOP\&AP-DOA algorithms at each SNR. It is seen that the number of iterations of AOP\&AP-DOA is the smallest, while the number of iterations of RELAX and AOP-DOA are approximately the same though the latter is slightly smaller. Particularly, the iteration number of PSBL decreases fast when the SNR increases, and that's the reason the CPU time of PSBL fluctuates (the complexity per iteration of PSBL is identical [27]). These phenomena are common in the following simulations, hence the curve of the number of iterations is omitted, and the similar phenomena are not explained repeatedly.

Figure 4 considers two uncorrelated signals with different angular separations. We assume $\Theta_{1}=5.37^{\circ}$ and $\Theta_{2}=5.37^{\circ}-\Delta$, where $\Delta$ denotes the source separation and it ranges from $2^{\circ}$ to $24^{\circ}$. The SNR is fixed at $10 \mathrm{~dB}$. It is seen that the proposed AOP-DOA and AOP\&AP-DOA have a distinct advantage against spatially adjacent signals, and both are high resolution algorithms. Compared with APMLE, the proposed algorithms exhibit the similar estimation precision but consume much less running time. Compared with PSBL and RELAX, the proposed algorithms exhibit a lower RMSE when the angular separation is small, and the time usage of the proposed algorithms decreases rapidly when the angular separation largely increases. A large angular separation may lead to a faster convergence speed of an iterative algorithm, but it is not the main factor that determines the computational complexity of MUSIC and $l_{1}$-SVD (cf. [6], [45], [23]). The CPU time of MUSIC and $l_{1}-$ SVD has tiny changes for different angular separations. Hence, as the angular separation becomes largely, the time usage of the proposed AOP-DOA could be lower than that of the MUSIC and $l_{1}$-SVD.

Both Fig. 5 and Fig. 7 consider the case of correlated signals. We assume two signals impinge on the array from directions $\left[-15.12^{\circ}, 5.37^{\circ}\right]$. In Fig. 5, the correlation coefficient of the signals is set to 0.5 , and we vary the SNR from $-15 \mathrm{~dB}$ to $20 \mathrm{~dB}$. In Fig. 7 , the SNR is fixed at $15 \mathrm{~dB}$, but we vary the correlation coefficient of the signals from 0 to 1 . The simulation results illustrated in Fig. 5 and Fig. 7 


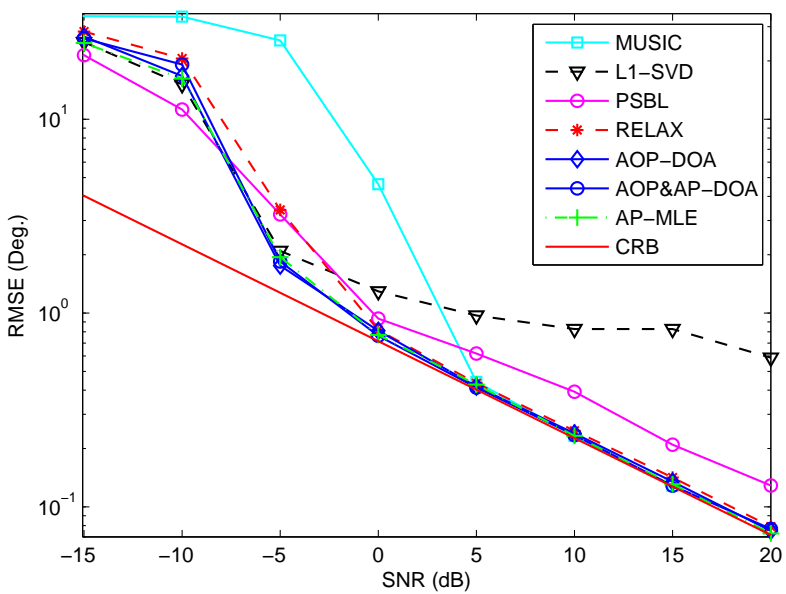

(a) RMSE versus SNR

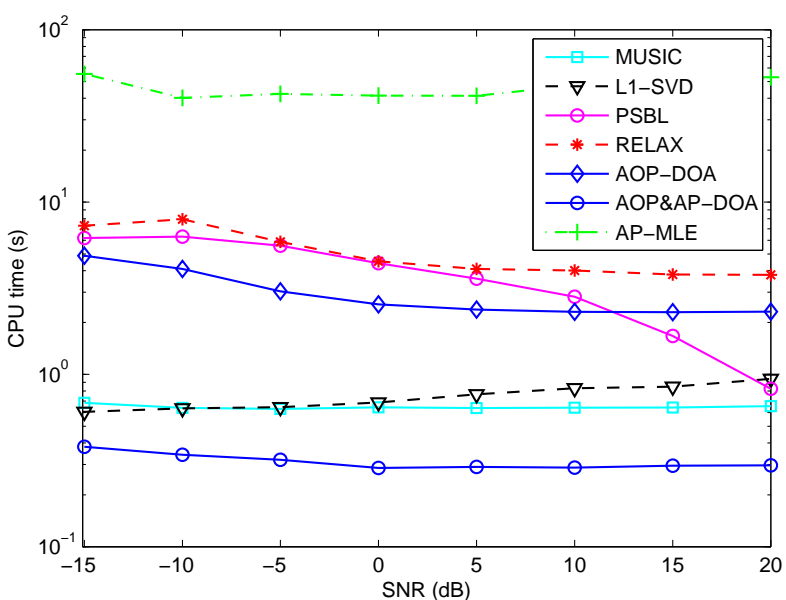

(b) CPU time versus SNR

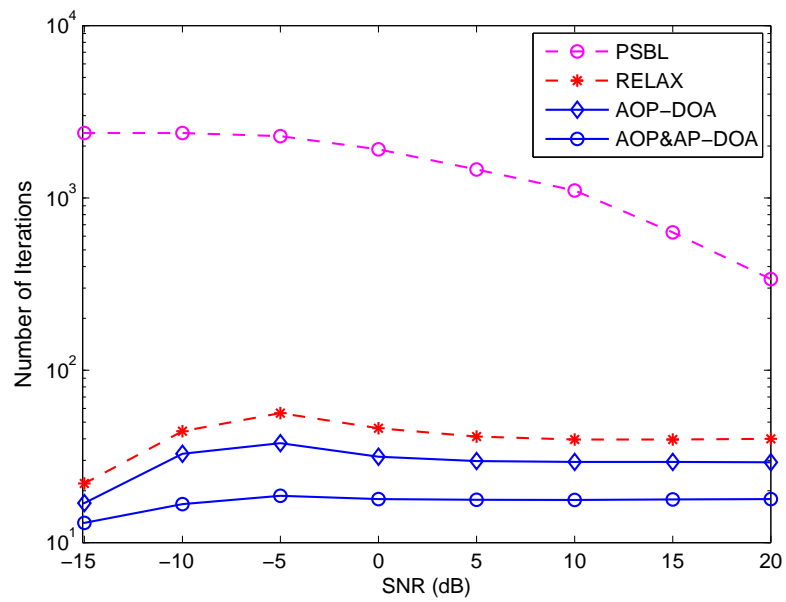

(c) Number of iterations at each SNR

Fig. 3. RMSE and CPU time comparison for two uncorrelated sources impinging from $\left[-5.12^{\circ}, 5.37^{\circ}\right]$

show that the proposed AOP-DOA and AOP\&AP-DOA can achieve satisfactory accuracies when the signals are partially or fully correlated. The DOA estimation performance of each algorithm deteriorates along with the increased correlation coefficient, and the MUSIC fails when the signals

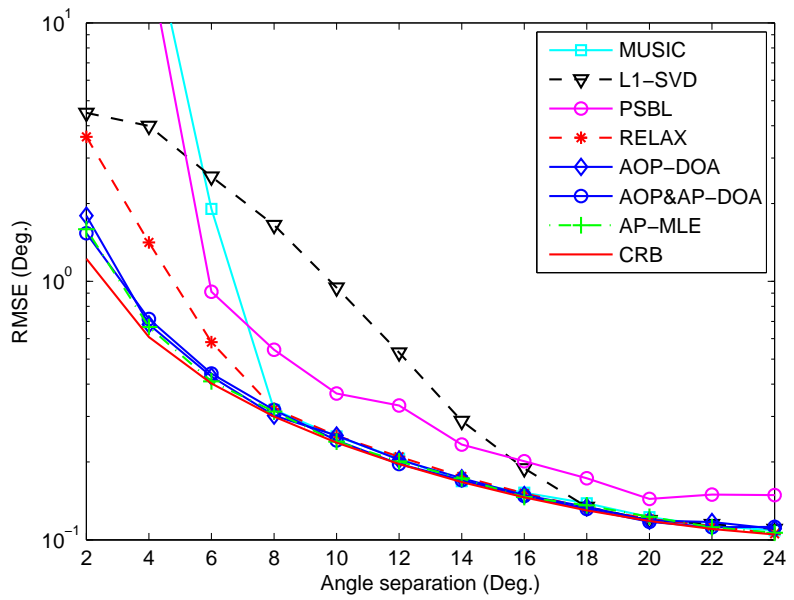

(a) RMSE versus angular separation

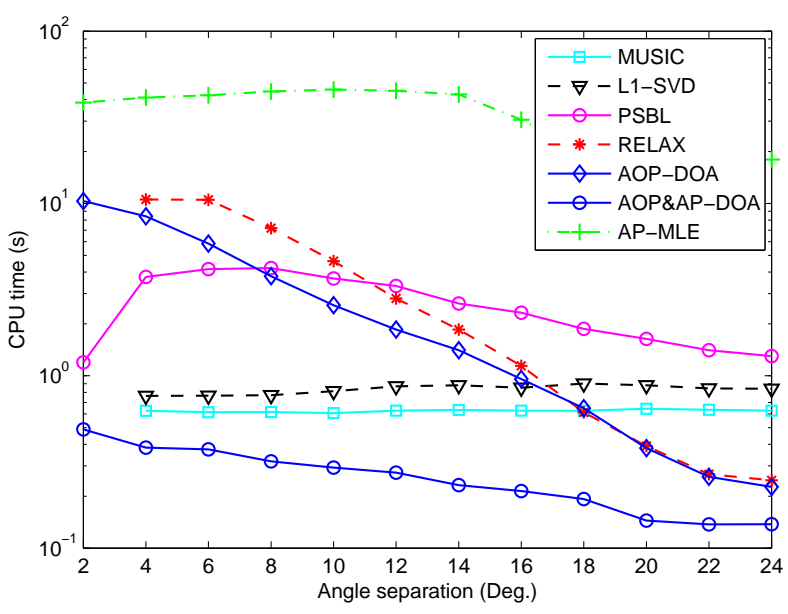

(b) CPU time versus angular separation

Fig. 4. RMSE and CPU time comparison for two uncorrelated sources impinging from $\left[5.37^{\circ}, 5.37^{\circ}-\Delta\right]$, where the angular separation $\Delta$ ranges from $2^{\circ}$ to $24^{\circ}$ and the SNR is fixed at $10 \mathrm{~dB}$.

are coherent. But the proposed AOP-DOA and AOP\&APDOA, as well as AP-MLE and RELAX, are able to coincide well with the CRB, and they could have a smaller RMSE than PSBL and $l_{1}$-SVD. In addition, the proposed two algorithms have a much lower computational cost when compared with AP-MLE, even if the sources are correlated. The increased correlation coefficient of signals makes AP-MLE, RELAX, AOP-DOA and AOP\&AP-DOA need more iterations (running time) to achieve convergence, whereas the complexities of MUSIC, $l_{1}$-SVD and PSBL are insensitive to the variations of the correlation property. Hence the MUSIC, $l_{1}-\mathrm{SVD}$ and PSBL show a stable running time, and this causes their CPU time curves to have intersections.

Figure 6 considers the DOA estimation for a mixture of uncorrelated and coherent signals. The incident signals are a group of two coherent signals from $\left[-15.12^{\circ}, 5.37^{\circ}\right]$ and an uncorrelated signal from $42.95^{\circ}$. The SNR ranges from $-5 \mathrm{~dB}$ to $20 \mathrm{~dB}$. The simulation results depicted in Fig. 6 


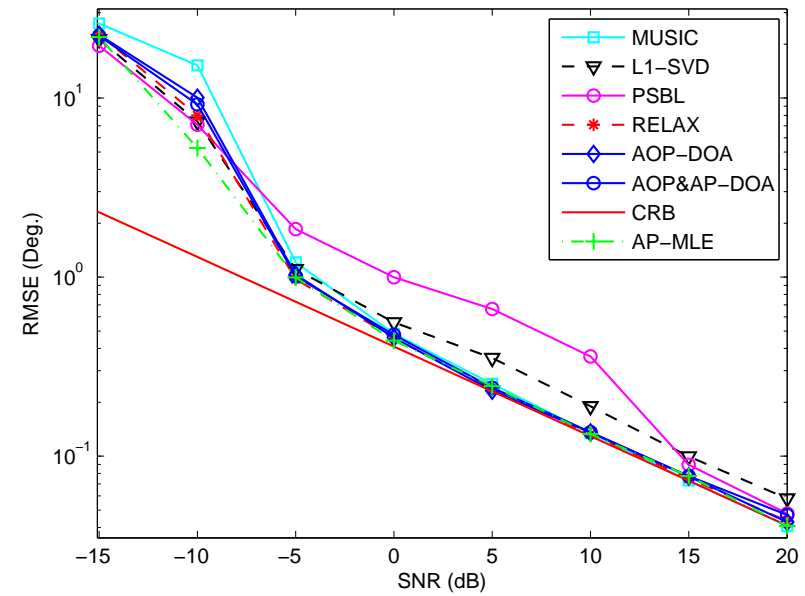

(a) RMSE versus SNR

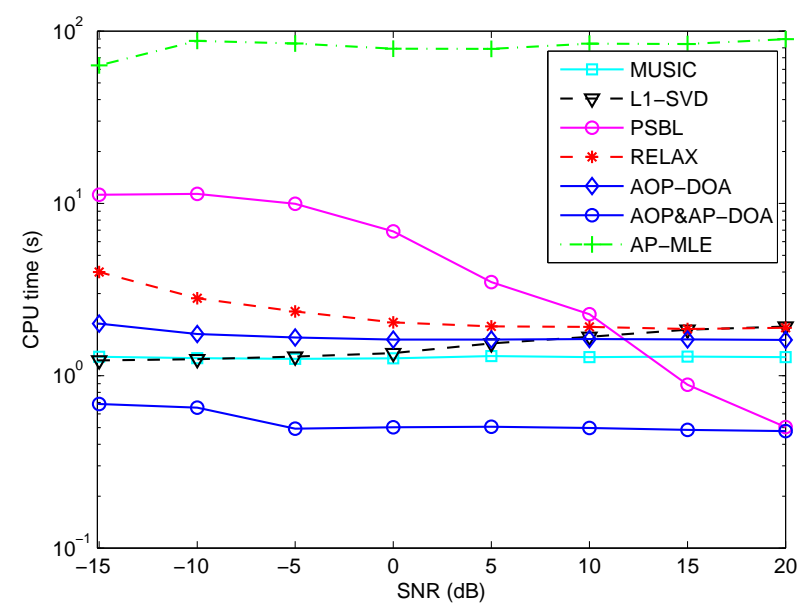

(b) CPU time versus SNR

Fig. 5. RMSE and CPU time comparison for two partially correlated sources impinging from $\left[-15.12^{\circ}, 5.37^{\circ}\right]$, where the correlation coefficient of the signals is set to 0.5 .

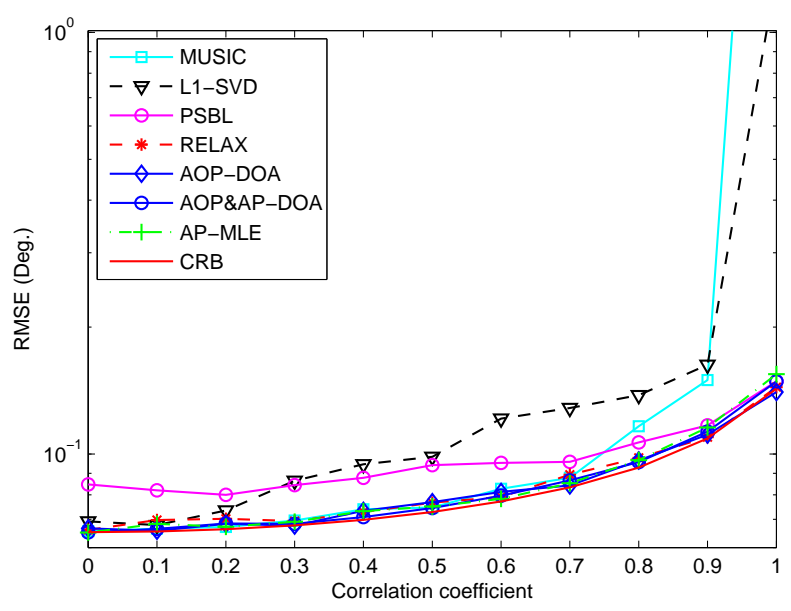

(a) RMSE versus correlation coefficient

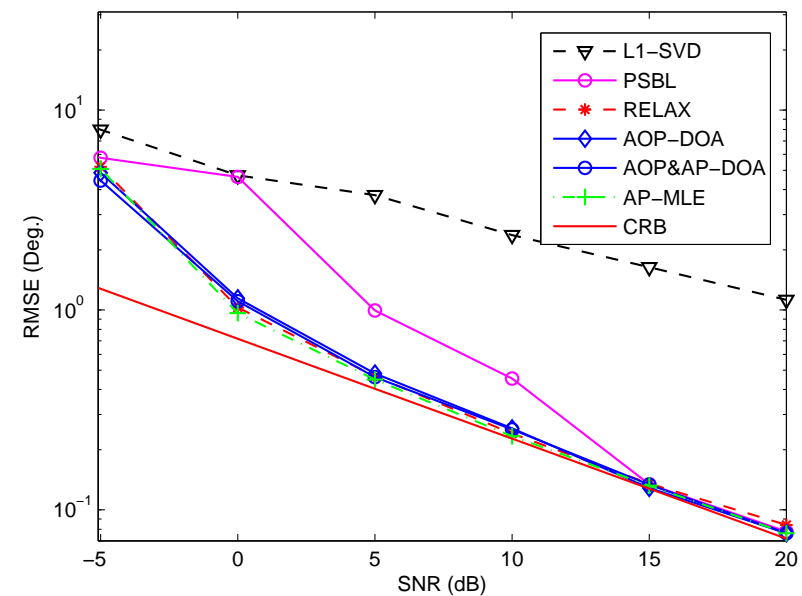

(a) RMSE versus SNR

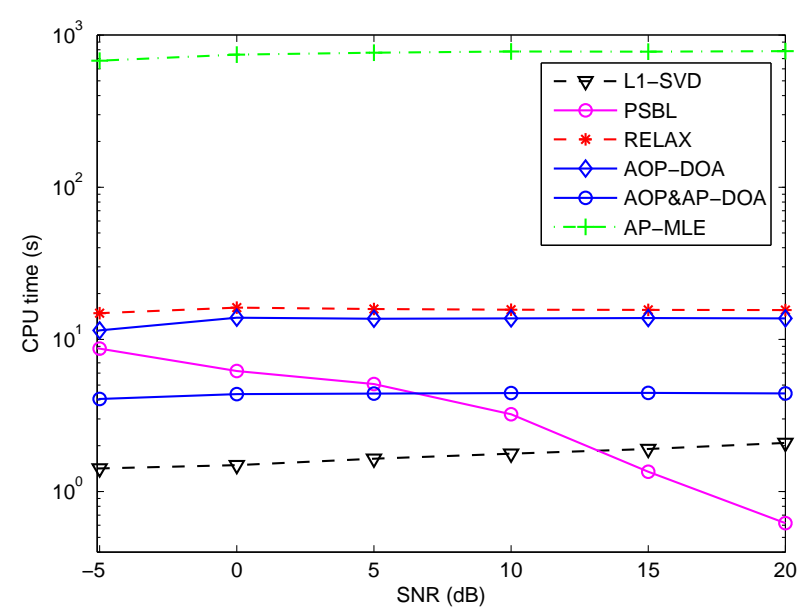

(b) CPU time versus SNR

Fig. 6. RMSE and CPU time comparison for a mixture of uncorrelated and coherent signals, where a group of two coherent signals from $\left[-15.12^{\circ}, 5.37^{\circ}\right]$ and an uncorrelated signal from $42.95^{\circ}$.

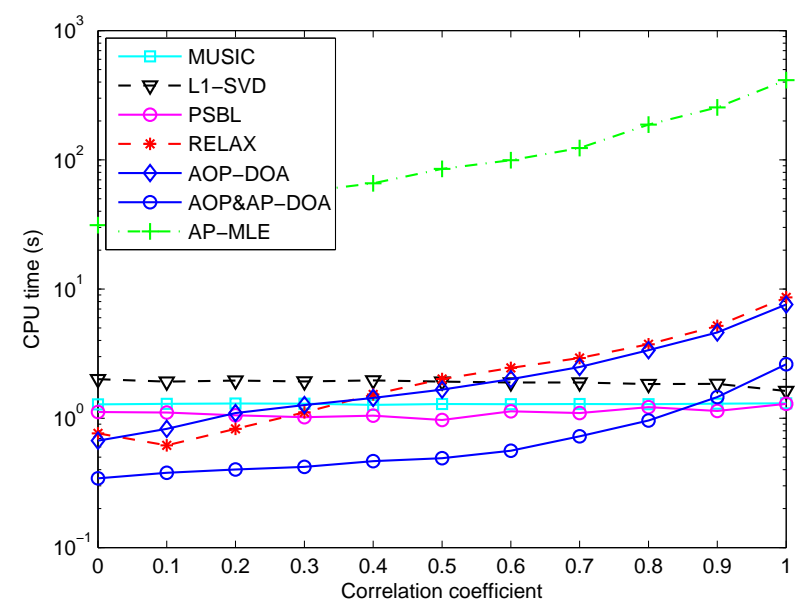

(b) CPU time versus correlation coefficient

Fig. 7. RMSE and CPU time comparison for two sources impinging from $\left[-15.12^{\circ}, 5.37^{\circ}\right]$, where the SNR is fixed at $15 \mathrm{~dB}$, but the sources' correlation coefficient varies. 
show that the proposed AOP-DOA and AOP\&AP-DOA algorithms, as well as the RELAX and AP-MLE, exhibit a satisfying estimation performance when uncorrelated and fully correlated signals coexist (remark that the MUSIC algorithm fails in this case, thus it is omitted here). On the whole, when compared with PSBL, the proposed algorithms have a better estimation performance and a more stable computational load under different SNR. Compared with $l_{1}$-SVD, the proposed algorithms can have a more superior estimation performance, although their computational loads are slightly higher in this scenario. In addition, as we have already verified in the previous simulations, the proposed AOP-DOA and AOP\&AP-DOA have a much lower computational load when compared with AP-MLE.

\subsection{Probability of Success Comparison}

This subsection intends to evaluate the success probabilities of the proposed AOP-DOA and AOP\&AP-DOA algorithms. The DOA estimation of $K$ signals is considered successful if and only if $\left|\hat{\Theta}_{1}-\Theta_{1}\right|,\left|\hat{\Theta}_{2}-\Theta_{2}\right|, \cdots,\left|\hat{\Theta}_{K}-\Theta_{K}\right|$ are less than a user-selected parameter $\epsilon$, which is set to be $1^{\circ}$ in the following simulations.

Figure 8 considers two uncorrelated signals with different angular separations. The signals impinge on the array from $\Theta_{1}=5.37^{\circ}$ and $\Theta_{2}=5.37^{\circ}-\Delta$, and the source separation $\Delta$ ranges from $2^{\circ}$ to $20^{\circ}$. In addition, the SNR is fixed at $10 \mathrm{~dB}$.

Figure 9 considers three signals, where we assume a group of two correlated signals with correlation coefficient $r$ impinge on the array from $\left[-15.12^{\circ}, 5.37^{\circ}\right]$ while an uncorrelated signal impinges on the array from $42.95^{\circ}$. The correlation coefficient $r$ is set to 0.9 and 1 in Fig. 9(a) and Fig. 9(b), respectively. Other settings remain the same as the last experiment considered in Sec. 4.2.

Both Fig. 8 and Fig. 9 show that the proposed AOPDOA and AOP\&AP-DOA algorithms have a relatively higher success probability when compared with $l_{1}$-SVD, PSBL and MUSIC. In addition, the success probability of the proposed AOP\&AP-DOA is able to coincide well with that of the APMLE, and is not lower than that of the RELAX. Remark that when signals are coherent, i.e., $r=1$, the RMSE of $l_{1}$-SVD is relatively higher than the threshold $\epsilon$ (cf. Fig. 6(a)). So the success probability of $l_{1}$-SVD is low. Meanwhile, the MUSIC is inefficient for identifying DOAs of coherent signals, hence it fails.

Figure 10 tests the proposed AOP-DOA and AOP\&APDOA algorithms by using a uniform circular array (UCA), where we consider azimuth-only DOA estimation and assume a group of two coherent signals impinging from $\left[-15.12^{\circ}\right.$, $\left.5.37^{\circ}\right]$ and an uncorrelated signal impinging from $42.95^{\circ}$. The SNR ranges form $-15 \mathrm{~dB}$ to $20 \mathrm{~dB}$. $M=15$ and the UCA is considered with two different radii. It can be seen that the proposed two algorithms are applicable to UCA, and this is because they have no limitation on antenna array geometry.

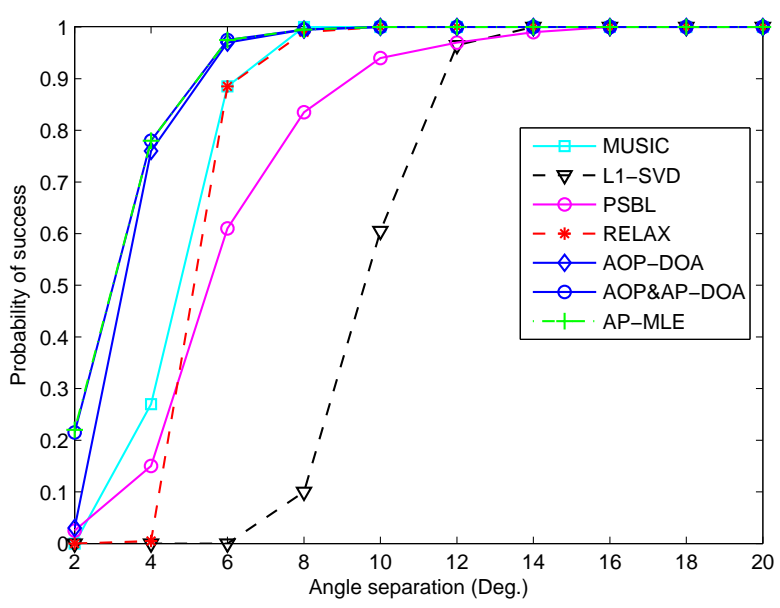

Fig. 8. Probability of success comparison for two uncorrelated signals impinging from $\left[5.37^{\circ}, 5.37^{\circ}-\Delta\right]$, where $\Delta$ ranges from $2^{\circ}$ to $20^{\circ}$ and the SNR is fixed at $10 \mathrm{~dB}$.

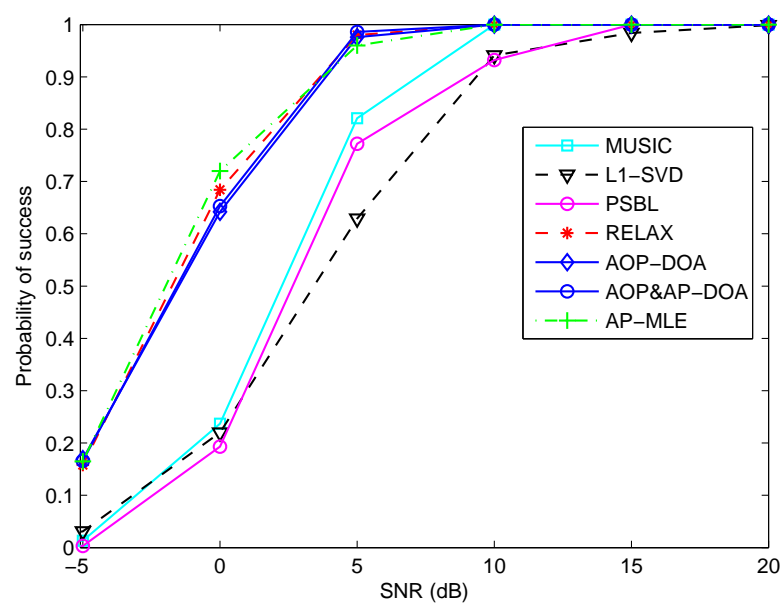

(a) Probability of success versus SNR when $r=0.9$

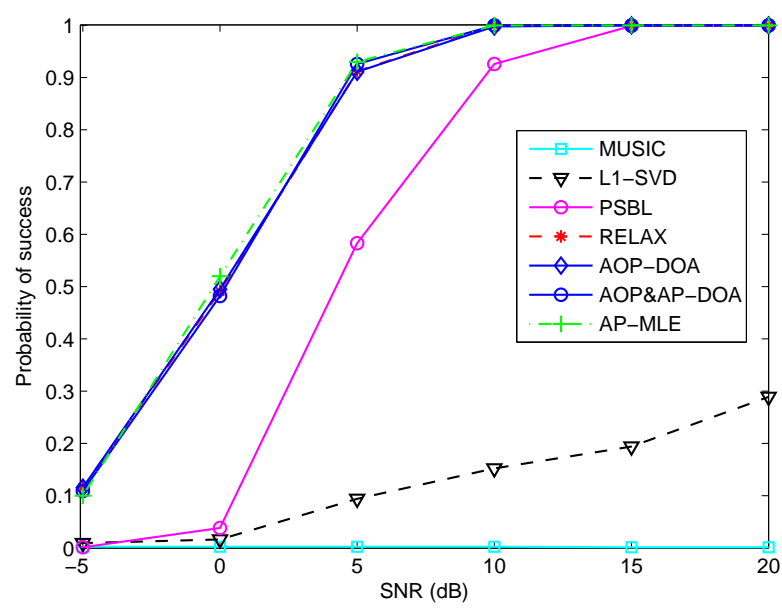

(b) Probability of success versus SNR when $r=1$

Fig. 9. Probability of success comparison for a mixture of uncorrelated and correlated signals, where a group of two correlated signals with correlation coefficient $r$ impinging from $\left[-15.12^{\circ}, 5.37^{\circ}\right]$ and an uncorrelated signal impinging from $42.95^{\circ}$. 


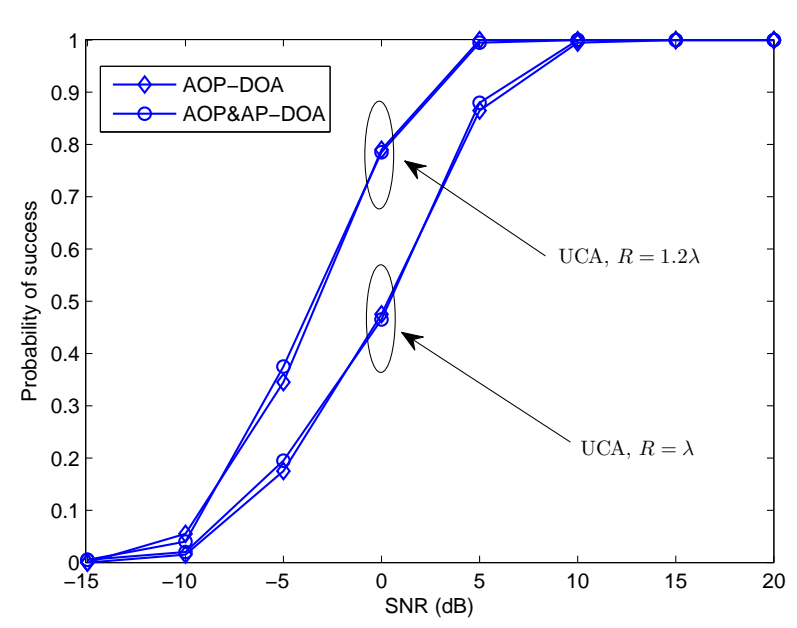

Fig. 10. Probability of success comparison of the proposed AOPDOA and AOP\&AP-DOA for a mixture of uncorrelated and coherent signals by using a UCA, where $R$ and $\lambda$ denote array radius and wavelength, respectively.

\section{Conclusion}

In this paper, a new iterative AOP-DOA algorithm is proposed for the DOA estimation of uncorrelated and correlated signals. To reduce the computational complexity of AOP-DOA, an algorithm named AOP\&AP-DOA, which is a combination of AOP-DOA and AP-MLE, is also proposed.

Both AOP-DOA and AOP\&AP-DOA are able to estimate DOAs of uncorrelated, partially correlated and coherent signals. The simulation results indicate that they exhibit excellent performance in terms of accuracy, success probability and complexity, when compared with MUSIC, $l_{1}$-SVD, PSBL, RELAX and AP-MLE. Firstly, for coherent signals, AOP-DOA has a distinct advantage over MUSIC in terms of DOA estimation since the latter algorithm is not applicable to this case. Secondly, compared with $l_{1}$-SVD and PSBL, AOPDOA presents better estimation accuracy within moderate to high SNR region and presents a relatively higher success probability in low SNR region. Thirdly, the complexities of AOP-DOA and RELAX are approximately presented at the same level, but when the angular separation is small, for uncorrelated signals, the accuracy and success probability of AOP-DOA are higher than those of RELAX. Lastly, when compared with AP-MLE, the proposed AOP-DOA not only exhibits a much lower computational complexity but also shows competitive success probability and accuracy.

Compared with AOP-DOA, the AOP\&AP-DOA not only keeps the merits of AOP-AOP, but also can be implemented with a much lower computational cost when proper adaptive grid refinement strategy is applied. In addition, both AOP-DOA and AOP\&AP-DOA are not restricted by the array geometry. Extensive experiments have been undertaken to verify our analysis and demonstrate the performance of our algorithms in cases of uncorrelated and correlated signals as compared to the existing ones. Note that only the white noise scenario is analyzed in this paper. Future work includes extension of the proposed algorithms to general noise environments [50], [20].

\section{Acknowledgments}

This work was supported in part by the National Natural Science Foundation of China (Grants No. 61171180 and No. 61371100) and the Fundamental Research Funds for the Central Universities (Grants No. HIT. MKSTISP. 201613 and No. HIT. MKSTISP. 2016 26).

\section{References}

[1] KRIM, H., VIBERG, M. Two decades of array signal processing research: the parametric approach. IEEE Signal Processing Magazine, 1996, vol. 13, no. 4, p. 67-94. DOI: $10.1109 / 79.526899$

[2] GAN, L., LUO, X. Direction-of-arrival estimation for uncorrelated and coherent signals in the presence of multipath propagation. IET Microwaves, Antennas and Propagation, 2013, vol. 7, no. 9, p. 746-753. DOI: 10.1049/iet-map.2012.0659

[3] STOICA, P., NEHORAI, A. Performance study of conditional and unconditional direction-of-arrival estimation. IEEE Transactions on Acoustics, Speech, and Signal Processing, 1990, vol. 38, no. 10, p. 1783-1795. DOI: $10.1109 / 29.60109$

[4] STOICA, P., SHARMAN, K. C. Maximum likelihood methods for direction-of-arrival estimation. IEEE Transactions on Acoustics, Speech, and Signal Processing, 1990, vol. 38, no. 7, p. 1132-1143. DOI: $10.1109 / 29.57542$

[5] SHEINVALD, J., WAX, M., WEISS, A. J. On maximumlikelihood localization of coherent signals. IEEE Transactions on Signal Processing, 1996, vol. 44, no. 10, p. 2475-2482. DOI: $10.1109 / 78.539032$

[6] SCHMIDT, R. O. Multiple emitter location and signal parameter estimation. IEEE Transactions on Antennas and Propagation, 1986, vol. 34 , no. 3 , p. 276-280. DOI: 10.1109/TAP.1986.1143830

[7] ROY, R., KAILATH, T. ESPRIT-estimation of signal parameters via rotational invariance techniques. IEEE Transactions on Acoustics, Speech, and Signal Processing, 1989, vol. 37, no. 7, p. 984-995. DOI: $10.1109 / 29.32276$

[8] XIN, J. M., SANO, A. Computationally efficient subspace-based method for direction-of-arrival estimation without eigendecomposition. IEEE Transactions on Signal Processing, 2004, vol. 52, no. 4, p. 876-893. DOI: 10.1109/TSP.2004.823469

[9] ZOLTOWSKI, M., HABER, F. A vector space approach to direction finding in a coherent multipath environment. IEEE Transactions on Antennas and Propagation, 1986, vol. 34, no. 9, p. 1069-1079. DOI: 10.1109/TAP.1986.1143956

[10] TAO, H., XIN, J., WANG, J., et al. Two-dimensional direction estimation for a mixture of noncoherent and coherent signals. IEEE Transactions on Signal Processing, 2015, vol. 63, no. 2, p. 318-333. DOI: 10.1109/TSP.2014.2369004

[11] LINEBARGER, D. A. Redundancy averaging with large arrays. IEEE Transactions on Signal Processing, 1993, vol. 41, no. 4, p. 17071710. DOI: $10.1109 / 78.212750$

[12] PILLAI, S. U., KWON, B. H. Forward/backward spatial smoothing techniques for coherent signal identification. IEEE Transactions on Acoustics, Speech, and Signal Processing, 1989, vol. 37, no. 1, p. 8-15. DOI: $10.1109 / 29.17496$

[13] WAX, M., SHEINVALD, J. Direction finding of coherent signals via spatial smoothing for uniform circular arrays. IEEE Transactions on Antennas and Propagation, 1994, vol. 42, no. 5, p. 613-620. DOI: $10.1109 / 8.299559$ 
[14] INDUKUMAR, K. C., REDDY, V. U. A note on redundancy averaging. IEEE Transactions on Signal Processing, 1992, vol. 40, no. 2 , p. 466-469. DOI: 10.1109/78.124962

[15] QIAN, C., HUANG, L., ZENG, W. J., et al. Direction-of-arrival estimation for coherent signals without knowledge of source number. IEEE Sensors Journal, 2014, vol. 14, no. 9, p. 3267-3273. DOI: $10.1109 /$ JSEN.2014.2327633

[16] RAHAMIM, D., TABRIKIAN, J., SHAVIT, R. Source localization using vector sensor array in a multipath environment. IEEE Transactions on Signal Processing, 2004, vol. 52, no. 11, p. 3096-3103. DOI: $10.1109 /$ TSP.2004.836456

[17] ZISKIND, I., WAX, M. Maximum likelihood localization of multiple sources by alternating projection. IEEE Transactions on Acoustics, Speech, and Signal Processing, 1988, vol. 36, no. 10, p. 1553-1560. DOI: $10.1109 / 29.7543$

[18] OH, S. K., UN, C. K. Efficient realisation of alternating projection algorithm for maximum likelihood direction finding. Electronics Letters, 1989, vol. 25, no. 20, p. 1325-1326. DOI: 10.1049/el:19890885

[19] VIBERG, M., OTTERSTEN, B., KAILATH, T. Detection and estimation in sensor arrays using weighted subspace fitting. IEEE Transactions on Signal Processing, 1991, vol. 39, no. 17, p. 2436-2449. DOI: 10.1109/78.97999

[20] LI, M. H., LU Y. L. Maximum likelihood DOA estimation in unknown colored noise fields. IEEE Transactions on Aerospace and Electronic Systems, 2008, vol. 44, no. 3, p. 1079-1090. DOI: $10.1109 /$ TAES.2008.4655365

[21] MASSA, A., ROCCA, P., OLIVERI, G. Compressive sensing in electromagnetics - a review. IEEE Antennas and Propagation Magazine, 2015, vol. 57, no. 1, p. 224-238. DOI: 10.1109/MAP.2015.2397092

[22] SAHOO, S. K., MAKUR, A. Signal recovery from random measurements via extended orthogonal matching pursuit. IEEE Transactions on Signal Processing, 2015, vol. 63, no. 10, p. 2572-2581. DOI: 10.1109/TSP.2015.2413384

[23] MALIOUTOV, D., CETIN, M., WILLSKY, A. S. A sparse signal reconstruction perspective for source localization with sensor arrays. IEEE Transactions on Signal Processing, 2005, vol. 53, no. 8 , p. 3010-3022. DOI: 10.1109/TSP.2005.850882

[24] YIN, J., CHEN, T. Direction-of-arrival estimation using a sparse representation of array covariance vectors. IEEE Transactions on Signal Processing, 2011, vol. 59, no. 9, p. 4489-4493. DOI: 10.1109/TSP.2011.2158425

[25] HYDER, M. M., MAHATA, K. Direction-of-arrival estimation using a mixed $l_{2,0}$ norm approximation. IEEE Transactions on Signal Processing, 2010, vol. 58, no. 9, p. 4646-4655. DOI: $10.1109 /$ TSP.2010.2050477

[26] CARLIN, M., ROCCA, P., OLIVERI, G., et al. Directions-of-arrival estimation through bayesian compressive sensing strategies. IEEE Transactions on Antennas and Propagation, 2013, vol. 61, no. 7, p. 3828-3838. DOI: 10.1109/TAP.2013.2256093

[27] WU, X., ZHU, W. P., YAN, J. Direction of arrival estimation for off-grid signals based on sparse bayesian learning. IEEE Sensors Journal, 2016, vol. 16, no. 7, p. 2004-2016. DOI: $10.1109 /$ JSEN.2015.2508059

[28] LI, J., ZHENG, D. M., STOICA, P. Angle and waveform estimation via RELAX. IEEE Transactions on Aerospace and Electronic Systems, 1997, vol. 33, no. 3, p. 1077-1087. DOI: 10.1109/7.599338

[29] YARDIBI, T., LI, J., STOICA, P., et al. Source localization and sensing: a nonparametric iterative adaptive approach based on weighted least squares. IEEE Transactions on Aerospace and Electronic Systems, 2010, vol. 46, no. 1, p. 425-443. DOI: 10.1109/TAES.2010.5417172
[30] BEHRENS, R. T., SCHARF, L. L. Signal processing applications of oblique projection operators. IEEE Transactions on Signal Processing, 1994, vol. 42, no. 6, p. 1413-1424. DOI: 10.1109/78.286957

[31] BOYER, R. Oblique projection for source estimation in a competitive environment: algorithm and statistical analysis. Signal Processing, 2009, vol. 89, no. 12, p. 2547-2554. DOI: $10.1016 /$ j.sigpro.2009.04.023

[32] SCHARF, L. L., MCCLOUD, M. L. Blind adaptation of zero forcing projections and oblique pseudo-inverses for subspace detection and estimation when interference dominates noise. IEEE Transactions on Signal Processing, 2002, vol. 50, no. 12, p. 2938-2946. DOI: $10.1109 / \mathrm{TSP} .2002 .805245$

[33] MCCLOUD, M. L., SCHARF, L. L. A new subspace identification algorithm for high-resolution DOA estimation. IEEE Transactions on Antennas and Propagation, 2002, vol. 50, no. 10, p. 1382-1390. DOI: $10.1109 /$ TAP.2002.805244

[34] BOYER, R., BOULEUX, G. Oblique projections for directionof-arrival estimation with prior knowledge. IEEE Transactions on Signal Processing, 2008, vol. 56, no. 4, p. 1374-1387. DOI: $10.1109 /$ TSP.2007.909348

[35] TAO, H., XIN, J. M., WANG, J. S., et al. Oblique projection based enumeration of mixed noncoherent and coherent narrowband signals. IEEE Transactions on Signal Processing, 2016, vol. 64, no. 16 , p. 4282-4295. DOI: 10.1109/TSP.2016.2548994

[36] TAO, H., XIN, J. M., WANG, J. S., et al. Two-dimensional direction estimation for a mixture of noncoherent and coherent signals. IEEE Transactions on Signal Processing, 2015, vol. 63, no. 2, p. 318-333. DOI: 10.1109/TSP.2014.2369004

[37] XU, X., YE, Z., Zhang Y., et al. A deflation approach to direction of arrival estimation for symmetric uniform linear array. IEEE Antennas and Wireless Propagation Letters, 2006, vol. 5, no. 1, p. 486-489. DOI: 10.1109/LAWP.2006.886304

[38] SI, W. J., ZHAO P. J., QU, Z. Y., et al. Real-valued DOA estimation for a mixture of uncorrelated and coherent sources via unitary transformation. Digital Signal Processing, 2016, vol. 58, p. 102-114. DOI: $10.1016 /$ j.dsp.2016.07.024

[39] CUI, Y., LIU, K., WANG, J. Direction-of-arrival estimation for coherent GPS signals based on oblique projection. Signal Processing, 2012, vol. 92, no. 1, p. 294-299. DOI: 10.1016/j.sigpro.2011.07.014

[40] XU, X., YE, Z., PENG, J. Method of direction-of-arrival estimation for uncorrelated, partially correlated and coherent sources. IET Microwaves, Antennas and Propagation, 2007, vol. 1, no. 4, p. 949-954. DOI: 10.1049/iet-map:20070006

[41] LU, Z., ZOUBIR, A. M. Source enumeration in array processing using a two-step test. IEEE Transactions on Signal Processing, 2015, vol. 63, no. 10, p. 2718-2727. DOI: 10.1109/TSP.2015.2414894

[42] HUANG, L., XIAO, Y., LIU, K., et al. Bayesian information criterion for source enumeration in large-scale adaptive antenna array. IEEE Transactions on Vehicular Technology, 2016, vol. 65, no. 5, p. 3018-3032. DOI: 10.1109/TVT.2015.2436060

[43] ROUBICEK, T., KRUZIK, M. Adaptive approximation algorithm for relaxed optimization problems. In Fast Solution of Discretized Optimization Problems. Birkhäuser (Basel), 2001, p. 242-254. DOI: 10.1007/978-3-0348-8233-0_18

[44] THEODORIDIS, S., SLAVAKIS, K., YAMADA, I. Adaptive learning in a world of projections. IEEE Signal Processing Magazine, 2011, vol. 28. no. 1, p. 97-123. DOI: 10.1109/MSP.2010.938752

[45] HURT, N. E. Maximum likelihood estimation and MUSIC in array localization signal processing: a review. Multidimensional Systems and Signal Processing, 1990, vol. 1, no. 3, p. 279-325. DOI: 10.1007/BF01812401 
[46] GOLUB, G. H., PEREYRA, V. The differentiation of pseudo-inverses and nonlinear least squares problems whose variables separate. SIAM Journal on Numerical Analysis, 1973, vol. 10, no. 2, p. 413-432. DOI: $10.1137 / 0710036$

[47] CIRRINCIONE, G., CIRRINCIONE, M., HERAULT, J., et al. The MCA EXIN neuron for the minor component analysis. IEEE Transactions on Neural Networks, 2002, vol. 13, no. 1, p. 160-187. DOI: $10.1109 / 72.977295$

[48] MANGASARIAN, O. L., WILD, E. W., Multisurface proximal support vector machine classification via generalized eigenvalues. IEEE Transactions on Pattern Analysis and Machine Intelligence, 2006, vol. 28, no. 1, p. 69-74. DOI: 10.1109/TPAMI.2006.17

[49] STURM, J. F. Using SeDuMi 1.02, a MATLAB toolbox for optimization over symmetric cones. Optimization Methods and Software, 1999, vol. 11, no. 1-4, p. 625-653. DOI: $10.1080 / 10556789908805766$

[50] LIAO, B., CHAN, S. C., HUANG, L., et al. Iterative methods for subspace and DOA estimation in nonuniform noise. IEEE Transactions on Signal Processing, 2016, vol. 64, no. 12, p. 3008-3020. DOI: $10.1109 /$ TSP.2016.2537265

\section{About the Authors ...}

Huijun HOU was born in Anhui, China in 1987. He received the M.S. degree in Information and Communication Engineering from Harbin Institute of Technology, Harbin, China in 2012. He is currently working towards the Ph.D. degree at Harbin Institute of Technology. His research interests include array signal processing and anti-jamming techniques.

Xingpeng MAO (corresponding author) was born in Liaoning, China in 1972. He received his Ph.D. degree from Harbin Institute of Technology, Harbin, China in 2004. He is currently a doctoral advisor and full professor in School of Electronic and Information Engineering, Harbin Institute of Technology, China. His research interests include radar systems and signal processing.

\section{Appendix A: Proof of (12)}

After AOP-DOA achieves convergence, it follows from (10) and (11) that

$$
s_{k}(t)=\mathbf{a}^{\dagger}\left(\Theta_{k}\right) \mathbf{y}_{k}(t) .
$$

Substituting (7) into (27), then we have

$$
s_{k}(t)=\mathbf{a}^{\dagger}\left(\Theta_{k}\right)\left(\mathbf{I}-\mathbf{E}_{\mathbf{B}_{k}, \mathbf{a}\left(\Theta_{k}\right)}\right) \mathbf{z}(t)
$$

Substituting (28) into (3), it follows that

$$
\begin{aligned}
& \frac{1}{L} \sum_{l=1}^{L}\left\|\mathbf{x}\left(t_{l}\right)-\sum_{k=1}^{K} \mathbf{a}\left(\Theta_{k}\right) s_{k}\left(t_{l}\right)\right\|^{2} \\
& =\frac{1}{L} \sum_{l=1}^{L}\left\|\mathbf{x}\left(t_{l}\right)-\sum_{k=1}^{K} \mathbf{P}_{\mathbf{a}\left(\Theta_{k}\right)}\left(\mathbf{I}-\mathbf{E}_{\mathbf{B}_{k}, \mathbf{a}\left(\Theta_{k}\right)}\right) \mathbf{z}\left(t_{l}\right)\right\|^{2}
\end{aligned}
$$

According to Appendix B, we have

$$
\mathbf{P}_{\mathbf{A}(\Theta)}=\sum_{k=1}^{K} \mathbf{P}_{\mathbf{a}\left(\Theta_{k}\right)}\left(\mathbf{I}-\mathbf{E}_{\mathbf{B}_{k}, \mathbf{a}\left(\Theta_{k}\right)}\right) \text {. }
$$

Consequently, (29) can be rewritten as
$\frac{1}{L} \sum_{l=1}^{L}\left\|\mathbf{x}\left(t_{l}\right)-\sum_{k=1}^{K} \mathbf{a}\left(\Theta_{k}\right) s_{k}\left(t_{l}\right)\right\|^{2}=\sum_{l=1}^{L} \frac{\left\|\mathbf{x}\left(t_{l}\right)-\mathbf{P}_{\mathbf{A}(\Theta)} \mathbf{z}\left(t_{l}\right)\right\|^{2}}{L}$

Substituting (1) into (31), then we have

$$
\begin{aligned}
& \frac{1}{L} \sum_{l=1}^{L}\left\|\mathbf{x}\left(t_{l}\right)-\sum_{k=1}^{K} \mathbf{a}\left(\Theta_{k}\right) s_{k}\left(t_{l}\right)\right\|^{2} \\
& =\frac{1}{L} \sum_{l=1}^{L}\left\|\mathbf{P}_{\mathbf{A}(\Theta)}^{\perp} \mathbf{z}\left(t_{l}\right)+\mathbf{n}\left(t_{l}\right)\right\|^{2}=\operatorname{tr}\left\{\hat{\mathbf{R}}_{n}\right\}=M \hat{\sigma}^{2}
\end{aligned}
$$

where $\hat{\mathbf{R}}_{n}=\frac{1}{L} \sum_{l=1}^{L} \mathbf{n}\left(t_{l}\right) \mathbf{n}^{\mathrm{H}}\left(t_{l}\right)=\hat{\sigma}^{2} \mathbf{I}$.

Substituting (8) into (31), then we have

$$
\begin{aligned}
& \frac{1}{L} \sum_{l=1}^{L}\left\|\mathbf{x}\left(t_{l}\right)-\sum_{k=1}^{K} \mathbf{a}\left(\Theta_{k}\right) s_{k}\left(t_{l}\right)\right\|^{2} \\
& =\frac{1}{L} \sum_{l=1}^{L}\left\|\mathbf{P}_{\mathbf{A}(\Theta)}^{\perp} \mathbf{x}\left(t_{l}\right)+\mathbf{P}_{\mathbf{A}(\Theta)} \mathbf{n}\left(t_{l}\right)\right\|^{2}=\operatorname{tr}\left\{\mathbf{P}_{\mathbf{A}(\Theta)}^{\perp} \hat{\mathbf{R}}_{x}\right\}+ \\
& \quad \operatorname{tr}\left\{\mathbf{P}_{\mathbf{A}(\Theta)} \hat{\mathbf{R}}_{n}\right\}=\operatorname{tr}\left\{\mathbf{P}_{\mathbf{A}(\Theta)}^{\perp} \hat{\mathbf{R}}_{x}\right\}+K \hat{\sigma}^{2}
\end{aligned}
$$

It follows from (32) and (33) that

$$
\begin{aligned}
& \frac{1}{(M-K) L} \sum_{l=1}^{L}\left\|\mathbf{x}\left(t_{l}\right)-\sum_{k=1}^{K} \mathbf{a}\left(\Theta_{k}\right) s_{k}\left(t_{l}\right)\right\|^{2}-\frac{K \hat{\sigma}^{2}}{M-K} \\
& =\frac{1}{M-K} \operatorname{tr}\left\{\mathbf{P}_{\mathbf{A}(\Theta)}^{\perp} \hat{\mathbf{R}}_{x}\right\}=\hat{\sigma}^{2} .
\end{aligned}
$$

\section{Appendix B: Proof of (30)}

It follows from (6) and [30] that

$$
\begin{aligned}
& \sum_{k=1}^{K} \mathbf{E}_{\mathbf{a}\left(\Theta_{k}\right), \mathbf{B}_{k}} \mathbf{A}(\boldsymbol{\Theta}) \\
& =\sum_{k=1}^{K}\left[\mathbf{E}_{\mathbf{a}\left(\Theta_{k}\right), \mathbf{B}_{k}} \mathbf{a}\left(\Theta_{1}\right), \cdots, \mathbf{E}_{\mathbf{a}\left(\Theta_{k}\right), \mathbf{B}_{k}} \mathbf{a}\left(\Theta_{K}\right)\right] \\
& =\mathbf{A}(\boldsymbol{\Theta}) .
\end{aligned}
$$

Thus, it holds that $\left(\sum_{k=1}^{K} \mathbf{E}_{\mathbf{a}\left(\Theta_{k}\right), \mathbf{B}_{k}}-\mathbf{I}\right) \mathbf{A}(\boldsymbol{\Theta})=\mathbf{0}$, and $\left(\sum_{k=1}^{K} \mathbf{E}_{\mathbf{a}\left(\Theta_{k}\right), \mathbf{B}_{k}}-\mathbf{I}\right) \mathbf{A}(\boldsymbol{\Theta}) \mathbf{A}^{\dagger}(\boldsymbol{\Theta})=\mathbf{0}$. Then,

$$
\mathbf{P}_{\mathbf{A}(\Theta)}=\sum_{k=1}^{K} \mathbf{E}_{\mathbf{a}\left(\Theta_{k}\right), \mathbf{B}_{k}} \mathbf{P}_{\mathbf{A}(\Theta)} .
$$

Note that $\mathbf{P}_{\mathbf{a}\left(\Theta_{k}\right)} \mathbf{P}_{\mathbf{A}(\Theta)}^{\perp}=\mathbf{0}, \mathbf{E}_{\mathbf{a}\left(\Theta_{k}\right), \mathbf{B}_{k}} \mathbf{E}_{\mathbf{B}_{k}, \mathbf{a}\left(\Theta_{k}\right)}=\mathbf{0}$ and $\mathbf{P}_{\mathbf{A}(\Theta)}=\mathbf{E}_{\mathbf{a}\left(\Theta_{k}\right), \mathbf{B}_{k}}+\mathbf{E}_{\mathbf{B}_{k}, \mathbf{a}\left(\Theta_{k}\right)}$. It follows that $\mathbf{E}_{\mathbf{a}\left(\Theta_{k}\right), \mathbf{B}_{k}}=$ $\mathbf{P}_{\mathbf{a}\left(\Theta_{k}\right)} \mathbf{E}_{\mathbf{a}\left(\Theta_{k}\right), \mathbf{B}_{k}}=\mathbf{P}_{\mathbf{a}\left(\Theta_{k}\right)}\left(\mathbf{I}-\mathbf{E}_{\mathbf{B}_{k}, \mathbf{a}\left(\Theta_{k}\right)}\right)$, and

$$
\mathbf{E}_{\mathbf{a}\left(\Theta_{k}\right), \mathbf{B}_{k}} \mathbf{P}_{\mathbf{A}(\Theta)}=\mathbf{E}_{\mathbf{a}\left(\Theta_{k}\right), \mathbf{B}_{k}} .
$$

Substituting (37) into (36), then

$$
\mathbf{P}_{\mathbf{A}(\Theta)}=\sum_{k=1}^{K} \mathbf{E}_{\mathbf{a}\left(\Theta_{k}\right), \mathbf{B}_{k}}=\sum_{k=1}^{K} \mathbf{P}_{\mathbf{a}\left(\Theta_{k}\right)}\left(\mathbf{I}-\mathbf{E}_{\mathbf{B}_{k}, \mathbf{a}\left(\Theta_{k}\right)}\right) .
$$

